WHC-SD-WM-TI-664, Rev. 0

\title{
WHC-SD-WM-TI-664
}

\section{EVALUATION OF FTIR-BASED ANALYTICAL METHODS FOR THE ANALYSIS OF SIMULATED WASTES}

\author{
T. V. Rebagay \\ R. J. Cash \\ D. A. Dodd \\ L. L. Lockrem \\ J. E. Meacham \\ W. D. Winkelman
}

Westinghouse Hanford Company

Richland, Washington 99352 


\section{DISCLAIMER}

\section{Portions of this document may be illegible in electronic image products. Images are produced from the best available original document.}


2. Title

EVALUATION OF FTIR-BASED ANALYTICAL METHODS FOR THE ANALYSIS OF SIMULATED WASTES

5. Key Words

FTIR, Analytical Methods, Ferrocyanide

\section{APPROVED FOR Fimb $1 / 30 / 94$ PUBLIC RELEASE}

\section{Abstract}

Three FTIR-based analytical methods that have potential to characterize simulated waste tank materials have been evaluated. These include: (1) fiber optics, modular transfer optic using light guides equipped with noncontact sampling peripherals, and (3) photoacoustic spectroscopy.

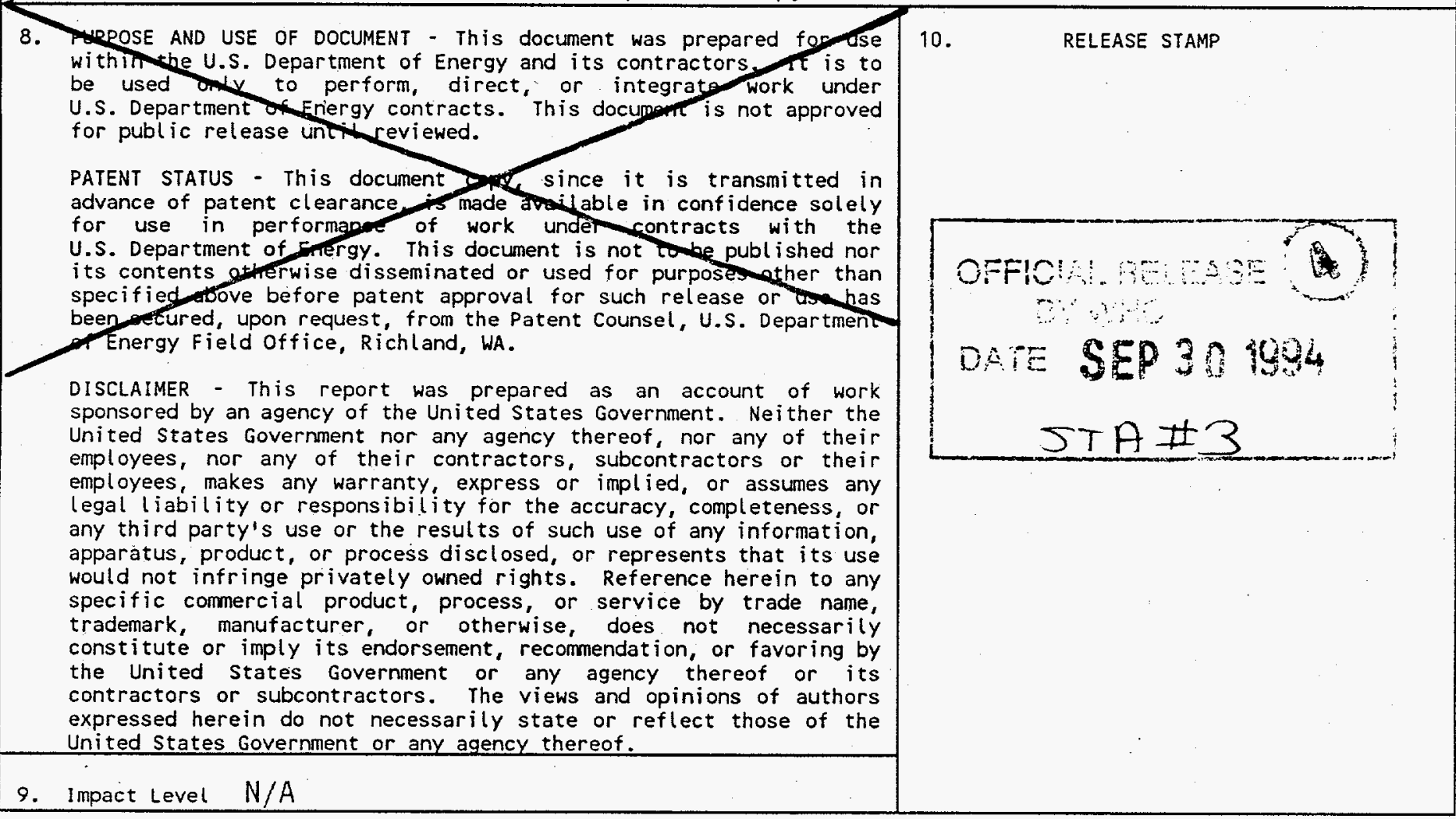




\section{RELEASE AUTHORIZATION}

Document Number: WHC-SD-WM-TI-664, REV 0

Document Title: $\quad$ EVALUATION OF FTIR-BASED ANALYTICAL METHODS FOR THE ANALYSIS OF SIMULATED WASTES

Release Date: $\quad$ September 30,1994

* * * * * * * * * * * * *

This document was reviewed following the procedures described in WHC-CM-3-4 and is:

APPROVED FOR PUBLIC RELEASE

* * * * * * * * * * * * *

WHC Information Release Administration Specialist:

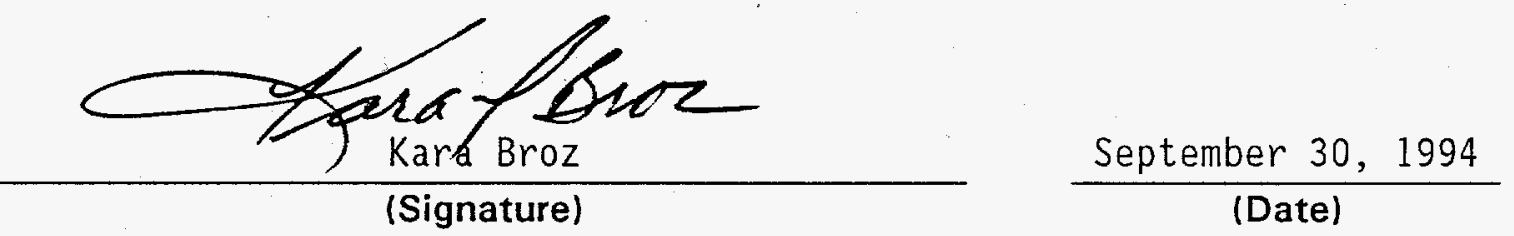


WHC-SD-WM-TI-664, Rev. 0

\title{
EVALUATION OF FTIR-BASED ANALYTICAL METHODS FOR THE ANALYSIS OF SIMULATED WASTES
}

\author{
T. V. Rebagay \\ R. J. Cash \\ D. A. Dodd \\ L. L. Lockrem \\ J. E. Meacham \\ W. D. Winkelman \\ Westinghouse Hanford Company
Richland, Washington 99352
}

\begin{abstract}
Three FTIR-based analytical methods that have potential to characterize simulated waste tank materials have been evaluated. These include: (1) fiber optics, (2) modular transfer optic using light guides equipped with noncontact sampling peripherals, and (3) photoacoustic spectroscopy. Pertinent instrumentation and experimental procedures for each method are described. The results show that the near-infrared (NIR) region of the infrared spectrum is the region of choice for the measurement of moisture in waste simulants. Differentiation of the NIR spectrum, as a preprocessing step, will improve the analytical result. Preliminary data indicate that prominent combination bands of water and the first overtone band of the ferrocyanide stretching vibration may be utilized to measure water and ferrocyanide species simultaneously. Both near-infrared and mid-infrared spectra must be collected, however, to measure ferrocyanide species unambiguously and accurately. For ease of sample handling and the potential for field or waste tank deployment, the FTIR-Fiber. Optic method is preferred over the other two methods. Modular transfer optic using light guides and photoacoustic spectroscopy may be used as backup systems and for the validation of the fiber optic data.
\end{abstract}


WHC-SD-WM-TI-664, Rev. 0

TABLE OF CONTENTS

$\begin{array}{lll}1.0 & \text { INTRODUCTION } & 1\end{array}$

2.0 FOURIER TRANSFORM INFRARED SPECTROSCOPY AND SYSTEM DESCRIPTION 2

2.1 Principles of FTIR Spectroscopy 2

2.1.1 Principle of the Michelson Interferometer 3

2.2 FTIR Spectrometry System 3

2.3 FTIR-Based Analytical Systems . 4

2.3.1 Fiber. Optics 4

2.3.1.1 Reflection Methods for Fiber Optics 4

2.3.1.2 Fiber Optic System Configuration 5

2.3.2 Modular Transfer Optics Using Light Guides 8

2.3.3 Photoacoustic Spectroscopy 9

3.0 EXPERIMENTAL PROCEDURES 11

3.1 Description of Materials and Sample Preparation 11

3.1.1 Reference Materials il

3.1.2 Waste Simulants 11

3.2 Spectral Data Acquisition 11

3.2.1 Evanescent Field Absorbance Sensor (EFAS) Method 11

3.2.2 Diffuse/Specular Reflectance Spectra Collection 14

3.2.3 Photoacoustic Spectra Measurement 14

3.2.4 Modular Transfer Optic Using Light Guides 15

3.2.5 Spectral Data Manipulation 15

$\begin{array}{lll}4.0 & \text { RESULTS AND DISCUSSION } & 17\end{array}$

$\begin{array}{lll}4.1 & \text { Fiber Optics Results } & 17\end{array}$

4.1.1 Evanescent Field Absorbance Sensor (EFAS) 17

4.1.2 Diffuse Reflectance/Specular Reflectance Results 20

4.2 Photoacoustic Spectroscopy Results 20

4.2.1 Reference Spectra of Some Ferrocyanide Compounds 20

4.2.2 Reference Spectra of Individual Simulant Components 21

4.2.3 Photoacoustic Spectral Data of Simulated Wastes 21

5.0 CONCLUSION 48

$\begin{array}{llr}6.0 & \text { REFERENCES } & 49\end{array}$ 
WHC-SD-WM-TI-664, Rev. 0

\section{LIST OF FIGURES}

Figure 2-1: Schematic of the Michelson Interferometer

Figure 2-2: Fiber Optics Accessory Layout

Figure 2-3: Types of Infrared Reflectances

Figure 2-4: Evanescent Field Absorbance Sensor

Figure 4-1: MIR Spectrum of Sludge on AgX Fiber 18

Figure 4-2: Comparison of MIR Sludge Spectra 18

Figure 4-3: Spectra of S7udge Using Light Pipes 18

Figure 4-4: NIR Spectra of Sludge on Silica Fiber 19

Figure 4-5: NIR Spectrum of Disodium Nickel Ferrocyanide (SRL Probe) 19

Figure 4-6: NIR Spectra of U Plant (As Received) Using SRL Probe 23

Figure 4-7: Derivative Spectra of U Plant (As Received) 24

Figure 4-8: Derivative Spectra of SY-101 (As Received) 25

Figure 4-9: NIR Spectra of Dried In Farm Simulant (SRL Fiber Probe) 26

Figure 4-10: NIR Spectra of Dried T Plant Simulant (SRL Fiber Probe) 27

Figure 4-11: NIR Spectra of Dried U Plant Simulant (SRL Fiber Probe) 28

Figure 4-12: NIR and MIR Spectra of Disodium Nickel Ferrocyanide 29

Figure 4-13: NIR and MIR Spectra of Sodium Ferrocyanide (PAS) 30

Figure 4-14: NIR and MIR Spectra of Potassium Ferrocyanide (PAS) 31

Figure 4-15: NIR and MIR Spectra of Nickel Ferrocyanide (PAS) 32

Figure 4-16: NIR and MIR Spectra of Magnesium Ferrocyanide (PAS) 33

Figure 4-17: NIR and MIR Spectra of Zinc Ferrocyanide (Photoacoustic) 34

Figure 4-18: NIR and MIR Spectra of Sodium Nitrate (PAS) 35

Figure 4-19: NIR and MIR Spectra of Sodium Nitrite (PAS) 36

Figure 4-20: NIR and MIR Spectra of Sodium Sulfate (PAS) 37

Figure 4-21: NIR and MIR Spectra of Ferrous Ammonium Sulfate (PAS) 38

Figure 4-22: NIR and MIR Spectra of Sodium Monohydrogen Phosphate (PAS) 39

Figure 4-23: NIR and MIR Spectra of Sulfamic Acid (PAS) 40

Figure 4-24: NIR Spectrum of Ammonium Sulfate (PAS) 41

Figure 4-25: NIR Spectrum of Potassium Phosphate (PAS) 41

Figure 4-26: NIR Spectrum of Potassium Sulfate (PAS) 42

Figure 4-27: NIR-PAS Spectrum of EDTA (Sodium Salt) • 42

Figure 4-28: NIR and MIR Spectra of BY104 (PAS) 43

Figure 4-29: NIR and MIR Spectra of In Farm Simulant (PAS) 44

Figure 4-30: NIR and MIR Spectra of SY101 Simulant (PAS) 45

Figure 4-31: NIR and MIR Spectra of T Plant Simulant (PAS) 46

Figure 4-32: NIR and MIR Spectra of U Plant Simulant (PAS) 47

\section{LIST OF TABLES}

Table 3-1: Infrared Reference Materials

Table 3-2: Simulated Waste Tank Wastes 
WHC-SD-WM-TI-664, Rev.0

\subsection{INTRODUCTION}

Fourier transform infrared (FTIR) spectroscopy provides an extremely sensitive probe of the molecular structure of materials. It is nondestructive, requires very little or no sample preparation, and offers a combination of broad spectral range $\left(15,000 \mathrm{~cm}^{-1}\right.$ to $\left.10 \mathrm{~cm}^{-1}\right)$ with relatively high spectral resolution, fast data acquisition times, and potential application over a wide range of time scales. For this reason, FTIR is an efficient and costeffective means to identify and quantify Hanford Site mixed wastes. However, candidate FTIR-based methods must be optimized for safety consideration of both equipment and personne?.

Sampling and chemical characterization of simulated and actual waste tank wastes are in progress at the Hanford Site. Because of the highly radioactive nature of actual Hanford tank waste, non-radioactive waste simulants mimicking actual tank wastes are used to provide an initial basis for identifying and quantifying realistic waste tank safety concerns. Emphasis has been placed on new or existing FTIR-based systems with potential for field or tank deployment to perform in situ remote waste characterization.

The validity of any method for mixed waste characterization must be demonstrated in terms of (1) its qualitative aspect and (2) its quantitative aspect. The objective of this investigation is to explore the qualitative aspect using waste simulants. Future efforts will focus on the quantitative aspect and its application to actual wastes.

A strategy was designed and developed to provide measurement information from which technical decisions could be derived. A survey of several available infrared measurement techniques was conducted before capital funding was expended, time was committed, and expenses incurred. A direct comparison of capabilities for each potential infrared system was performed by cursory examination of selected waste simulant samples using the vendor's infrared system at the vendor's laboratory. Results were evaluated which formed the basis for deciding on the best available system for Hanford Site waste characterization.

In this report, three FTIR-based analytical methods that showed potential to characterize simulated waste tank waste materials have been assessed. These include: (1) fiber optics, (2) modular transfer optic using light guides equipped with non-contact sampling peripherals, and (3) photoacoustic spectroscopy. Each method is described and the preliminary results of analyses on waste simulants are discussed. 
WHC-SD-WM-TI-664, Rev. 0

\subsection{FOURIER TRANSFORM INFRARED (FTIR) SPECTROSCOPY AND SYSTEM DESCRIPTION}

\subsection{Principles of FTIR Spectroscopy}

The infrared region of the electromagnetic spectrum lies between the visible and microwave regions. The infrared region may be divided into three regions: (1) near-infrared (NIR) from $13,300 \mathrm{~cm}^{-}$to $4000 \mathrm{~cm}^{-1}$; (2) mid-infrared (MIR) from $4000 \mathrm{~cm}^{-1}$ to $400 \mathrm{~cm}^{-1}$; and (3) far-infrared (FIR) from $400 \mathrm{~cm}^{-1}$ to $10 \mathrm{~cm}^{-1}$.

When a molecule absorbs infrared radiation of the appropriate frequency, it is excited from one vibrational or rotational level to another. A plot of the energy absorbed versus the frequency produces the absorption spectrum of the molecule. There are two types of infrared instruments that can be used to record the absorption spectrum of the molecule: (1) classical dispersive spectrometer and (2). FTIR spectrometer. Only the principle of FTIR is discussed in this report.

The main component of an FTIR spectrometer is the Michelson interferometer. In principle, an interferometer has several basic advantages over a classical dispersive spectrometer (Perkin Elmer, 1992). These advantages are:

- Multiplex Advantage- A complete spectrum can be obtained very rapidly and many scans can be averaged in the time taken for a single scan of a dispersive spectrometer.

- Throughput Advantage- For the same resolution, the energy throughput in an interferometer can be higher than a dispersive spectrometer where it is restricted by the slits.

- Connes Advantage- The frequency scale of an interferometer is derived from a helium-neon laser that acts as an internal reference for each scan. The frequency of this laser is known very accurately and is very stable. As a result, the frequency calibration of interferometers is more accurate and has longer term stability than the calibration of dispersive instruments.

o Negligible Stray Light-Because of the way in which the interferometer modulates each frequency, there is no direct equivalent of the stray light found in dispersive spectrometers.

- Constant Resolution- Resolution is similar at a11 wavelengths in the defined spectral range but the signal to noise ratio varies across the spectrum. FTIR instruments have a much higher optical throughput than dispersive instruments and do not use slits to define the resolution. Instead, the resolution is defined by the J-stop aperture size, which does not change during data 
WHC-SD-WM-TI-664, Rev. 0

collection. In dispersive instruments, throughput is typically optimized by adjusting the slit width during the scan.

o No discontinuities- As there are no grating or filter changes, there are no discontinuities in the spectrum.

\subsubsection{Principle of the Michelson Interferometer}

The schematic of the Michelson interferometer (Bio-Rad, 1993) is presented in Figure 2-1. The interferometer contains a fixed mirror, a movable mirror, and a beamsplitter. The beamsplitter transmits half of the incident radiation to the moving mirror and reflects the other half to the fixed mirror. The two beams are reflected by the mirrors back to the beamsplitter where they recombine.

When the fixed mirror and moving mirror are equidistant from the beamsplitter, all frequencies are in phase and recombine constructively. This position of zero path difference or zero retardation is where the centerburst occurs. As the position of the moving mirror changes, the two beams travel different distances before recombining. A pattern of constructive and destructive interferences is generated based on the position of the moving mirror and the frequency of the retardation. The intensity of the radiation varies in a complicated pattern and the output beam is modulated by the interferometer. This modulated output beam is then directed through the sample.compartment to the detector. At the detector, it generates a continuous electrical signal called an interferogram.

The moving mirror is driven at a constant velocity by a 1 inear motor under computer control to provide the varying optical path difference. A heliumneon laser operating at 632.8 nanometers generates a reference signal which enables the spectrometer electronics to sample the interferogram at precise intervals, generating a digital signal compatible with the computer. The computer converts the interferogram into a single-beam spectrum by a Fourier transform. When no sample is present, this gives a single beam spectrum (background spectrum), the overall shape of which is 1 argely determined by the characteristics of the beamsplitter and the source. Normally, interferometers operate by first recording this background and then ratioing the sample spectrum against it.

\subsection{FTIR Spectrometry System}

The FTIR spectrometry system used in this investigation is a Bio-Rad FTS $60 \mathrm{~A}$ spectrometer (Bio-Rad Analytical Instruments, Cambridge, MA) that features a dynamicaliy aligned interferometer with capability of spectral range changes through interchangeable beamsplitters. It includes a data system and an optical bench: The optical bench contains the interferometer and its associated electronics. The FTIR system has also been configured to accept 
WHC-SD-WM-TI-664, Rev. 0

visible and ultraviolet optical components.

A second sample compartment has been added to allow permanent installation of two primary sampling modes of the spectrometer: (1) a photoacoustic accessory and (2) a near-infrared fiber optic accessory. The photoacoustic and fiber optic probes are compatible for both infrared and visible spectral analysis, thereby enhancing the diagnostic information available from the spectrometer. This sample compartment has also been configured with its own external port that will support a modular transfer optics using light pipes.

\subsection{FTIR-Based Analytical Systems}

\subsubsection{Fiber Optics}

\subsubsection{Reflection Methods for Fiber Optics}

Fiber optics provide a nondestructive means of analyzing samples without the requirement that the samples be brought inside the sample chamber of the FTIR spectrometry system. This minimizes worker exposure to potentially toxic and radioactive environments. Un1ike other spectroscopic sampling arrangements, the fiber optic system is very flexible allowing analysis of infrared-active samples simply by choice of appropriate fiber type. Infrared-based fiber optics allows monitoring of moisture and other components of the waste remotely, safely, conveniently (in situ), and cost-effectively. Properly integrated to a waste tank surveillance system, it can provide real time assessment of the safety status of a waste tank.

When infrared rays impinge on a sample, the rays are either reflected, absorbed, transmitted, or scatterd. Mathematically, the rays' interaction with the sample can be represented by the following equation

$$
I_{0}=I_{r}+I_{a}+I_{t}+I_{s} \quad \text { Eq. } 1
$$

where $I_{0}$ is the intensity of the incident ray; $I_{r}$, the reflected ray; $I_{a}$, the absorbed ray; $I_{t}$, the transmitted ray; and $I_{s}$, the scattered ray. Thus the intensity of the absorbed ray, $I_{a}$, can be calculated by measuring $I_{r}, I_{t}$, and $I_{s}$.

Optically opaque and highly absorbing materials such as waste sludges are not amenable to transmission infrared methods. These sludges must be examined by reflection methods. Three possible reflection configurations in which an FTIR can be interfaced with fiber optics for characterization of sludges include: (1) attenuated total reflectance (ATR), (2) diffuse reflectance (DR), and specular reflectance (SR). The fiber optic probe can be made either as a single fiber or a bundle in a variety of fiber types, fiber lengths, and probe designs for reflection measurements. Unique requirements often dictate the use of application-specific optical fibers. 
WHC-SD-WM-TI-664, Rev. 0

The three types of reflections are sketched in Figure 2-3.

Attenuated Total Reflectance (ATR)- The attenuated total reflectance spectroscopic method of examining a sample uses the phenomenon whereby a light beam, inside an optical fiber, reflecting at a surface with a high refractive index, actually penetrates a short distance into an adjoining sample pressed against it. The fiber functions as an all-fiber absorption cell by exploiting the evanescent field interaction at the fiber/sample interface.

A property of the evanescent wave which makes ATR a powerful technique is that the intensity of the wave decays exponentially with distance from the surface of the fiber. This distance, which is on the order of micrometers, makes ATR generally insensitive to sample thickness, allowing for the analysis of thick or strongly absorbing samples. Previous work (Rebagay et.al, 1994) has shown that it is possible to characterize simulated wastes by this technique.

Diffuse Reflectance (DR)- Diffuse reflectance spectroscopy is used for samples that are also optically opaque and highly absorbing. In this method, incident infrared radiation is focused onto the inlet of the fiber(s). The infrared radiation penetrates one or more particles of the sample and is partially absorbed by the particle(s). The radiation that penetrates the particle(s) and exits from the surface of the particle(s) is reflected in all directions. This is then directed via the outlet end of the fiber toward a high sensitivity detector with the use of suitable focusing optics.

The primary function of the DR optical design is to efficiently collect that component of the reflected radiation that has penetrated the surface and interacted with the sample. A very important attribute of this spectroscopic technique is its extremely broad sampling concentration range, from highly dilute to neat.

Specular Reflectance (SR)-Specular reflection is a form of external reflectance in which the reflected radiation does not interact with sample molecules. The radiation reflects directly off the sample surface. Specular reflection occurs mostly on smooth, mirror-7ike surfaces. The analytical method is based on the spectral analysis of the dielectric reflection which occurs at the front surface of a sample. After recording a spectrum of a sample's reflectance, its absorbance spectrum can be calculated by means of a mathematical process called the Kramer-Kronig transform.

\subsubsection{Fiber Optic System Configuration}

The complete fiber optics system consists of an FTIR spectrometer (Bio-Rad FTS $60 A)$, a fiber optic accessory, a fiber optic probe, and a detector.

Fiber Optic Accessory- Figure 2-2 shows the layout of the commercial (Bio-Rad) near-infrared fiber optic accessory used in this study. It is mounted on a 
WHC-SD-WM-TI-664, Rev. 0

baseplate in the sample compartment of the FTIR spectrometer: A fixed flat mirror intercepts the beam from the IR source prior to reaching its normal focal point. It then passes through a focusing lens onto the entrance of the optical fiber probe. Two XYZ translational stages accurately position the fiber probe for efficient throughput. Swage fittings are used to mount the fiber into the XYZ stages. A second lens collects the transmitted infrared radiation and focuses it onto a detector (MCT) which is also integrated onto the baseplate in the sample compartment. The fiber optic accessory is designed to efficiently couple the optical beam of the spectrometer with the fiber optic probe which transfers the optical signal to the sample.

There are plans for utilizing NIR and MIR fiber optic probes sequentially. Since each fiber probe requires distinct detector and lens transfer optics for efficient performance, a second fiber optic accessory has been configured for the MIR region. This will support rapid interchange between the NIR and MIR measurements without the need for time consuming optical component swapping and realignment:

Fiber Optic Probes- Two types of fiber optic probes were fabricated: (1) ATR single fiber probes for the MIR and NIR regions and (2) DR and SR bifurcated bundle probes for the NIR region.

ATR Single Fiber Probes- Evanescent field absorbance sensors (EFAS) operating on the ATR principle were made from silver halide $(\mathrm{AgBr} / \mathrm{Cl})$ fiber and a polymer-clad, 10w-OH silica fiber for the MIR and NIR fiber optic probes, respectively. The fiber for the NIR-EFAS probe consisted of a $400-\mu \mathrm{m}$ outer diameter $10 \mathrm{w}-\mathrm{OH}$ fused silica core, a 500- $\mu \mathrm{m}$ outer diameter polymer cladding, and a $600-\mu \mathrm{m}$ outer diameter Nylon jacket. The center $12 \mathrm{~cm}$ of the 1-m polymer-clad silica fiber was stripped of its jacket and cladding. For the MIR-EFAS, a 1000- $\mu \mathrm{m}$ unclad $\mathrm{AgBr} / \mathrm{Cl}$ fiber was used. Each fiber probe was one meter long.

A sketch of the setup for both NIR and MIR EFAS systems is depicted in Figure 2-4. Each EFAS system consisted of the following: (1) FTIR spectrometer with spectral range covering both MIR and NIR regions, (2) a fiber positioner, (3) a stainless steel sampling trough, (4) a fiber coupler, and (5) a detector (MCT for MIR-EFAS or InSb for NIR-EFAS). Only the sampling trough was located inside a laboratory hood while the other components of the system were external to the hood.

NIR Bifurcated Bundle Fiber Probe- The NIR fiber optic probe was made by Savannah River Laboratory, Westinghouse Savannah River Site (Aiken, SC). It has a common leg and is bifurcated into two legs (one connected to the infrared source and the other to the detector) joined together in a zip cord configuration. The common leg contains a center fiber (detector fiber) surrounded by six other fibers (1ight source fibers). Each fiber has a 400- $\mu \mathrm{m}$

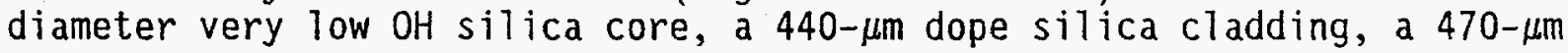


Schtmatic Of The Michelson Interferometer

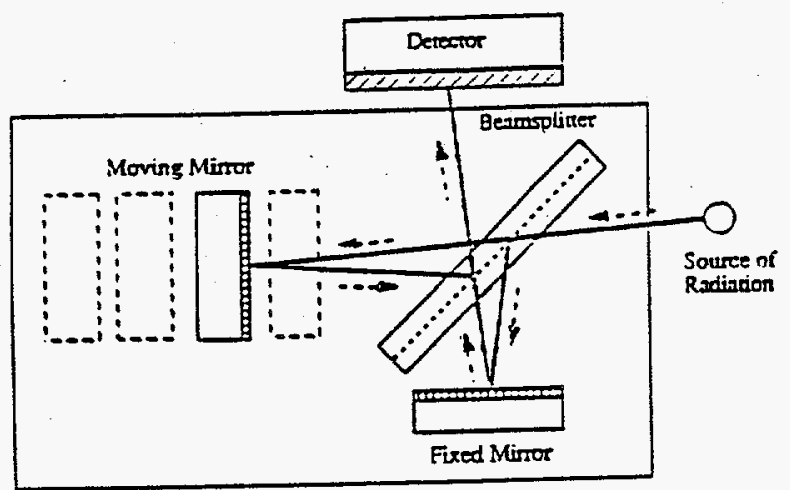

Figure 2-1. Schematic of the Michelson Interferometer
Fiber Optics Accessory Layout

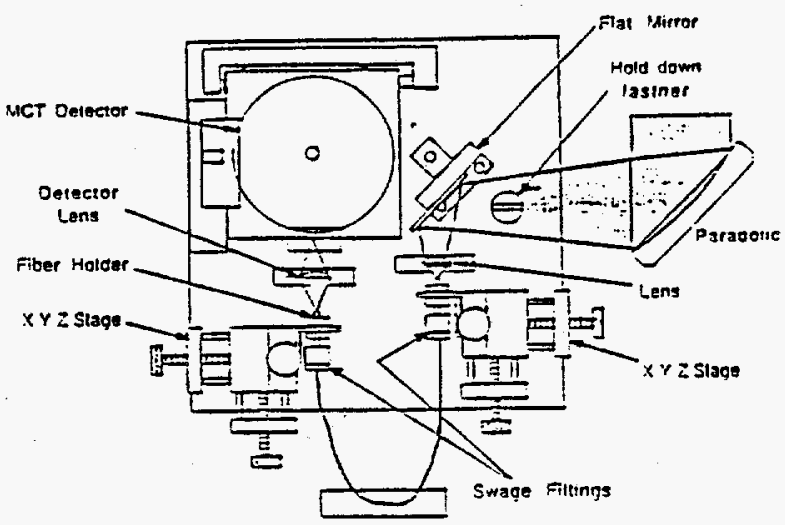

Figure 2-2. Fiber Optics Accessory Layout (NIR Mode)
Types of IR Reflectances

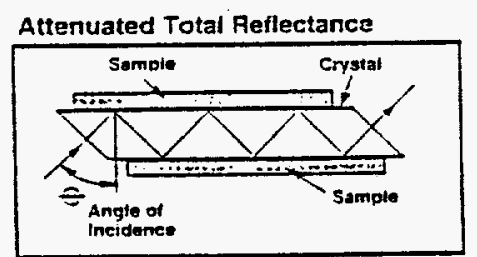

Diffuse Reflectance

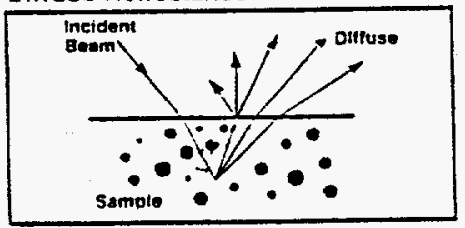

Specular Reflectance

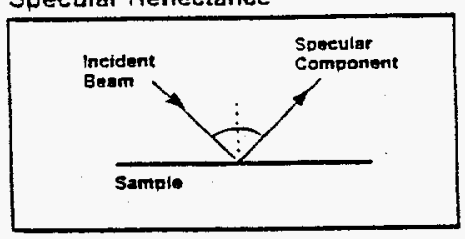

Evanescent Field Absorbance Sensor

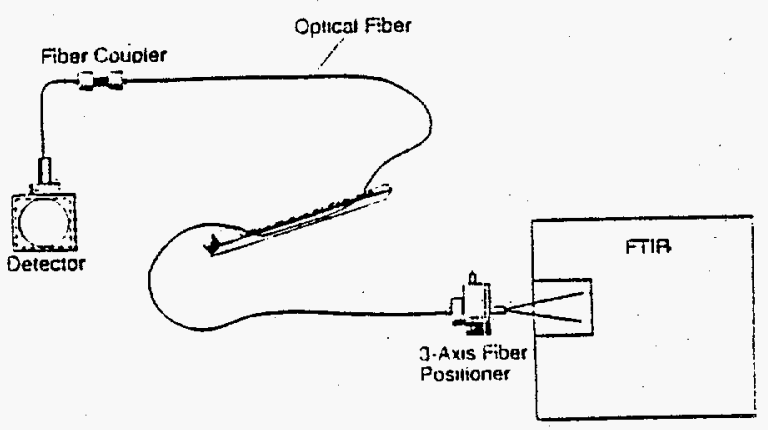

Figure 2-4. Evanescent Field Absorbance Sensor

Figure 2-3. Types of IR Reflections 
WHC-SD-WM-TI-664, Rev. 0

diameter polyimide buffer, and a 700- $\mu$ m polymer jacket. The polymer jacket was removed by immersion in acetone and mechanically stripped off using a wire stripper. The buffers of the common leg were molded together using an epoxy resin to form a bundle. The terminal of the common end was beveled toward the center fiber and then bonded to a 20-mil sapphire window using trimethoxych7oropropyl silane.

The light from each source fiber exited to the beveled surface, passed through the window, and then refracted toward the center fiber. The 1 ight transmitted through the window was scattered by the sample which may be in intimate contact with the window (diffuse reflectance mode) or about $1 \mathrm{~mm}$ away from the window (specular reflectance mode). The scattered light was collected by the detector fiber and directed to the detector. The bifurcated silica bundle was 5 meters long. This probe was then connected to the NIR fiber optic accessory inside the sample compartment of the FTIR spectrometer.

\subsubsection{Modular Transfer Optics Using Light Guides and Non-Contact Peripherals}

Optical wave guides (1ight guides) confine, guide, and provide a propagation path for the infrared light coming from the FTIR spectrometer. The transfer optics uses hollow metallic light guides to conduct the infrared beam over substantial distance $(20$ to $30 \mathrm{ft}$ ) without allowing beam spread.

A complete outboard FTIR modular transfer optic system consists of appropriate transfer optics, a sampling module (DR and SR samplers) and an infrared detector assembiy. The detector assembly includes adjustable focusing optics, a preamplifier, and a cable to transfer the electronic signal back to the spectrometry system. Once interfaced to the FTIR via the FTIR output port, operation of the system is similar to that of any FTIR system with conventional diffuse reflectance or specular reflectance sampler placed inside the FTIR sample chamber. The non-contact sampler (SR) used by the system is capable of eliminating polarization and orientation artifacts.

Like fiber optics, no sample preparation is necessary. The method requires sample sizes of only milligram amounts allowing analysis of radioactive waste samples to be performed in a laboratory hood. Spectra can be collected in both the MIR and NIR regions.

The performance of a modular transfer optic system has been demonstrated by the vendor and appeared to be satisfactory. The spectra of the test samples resembled those collected by conventional diffuse reflectance and specular reflectance samplers. Based on the vendor demonstration, this system may be used as a backup for the fiber optic system or for confirmation and/or validation of data derived from other techniques. Installation of a modular transfer optic assembly is scheduled in early FY' 95. 
WHC-SD-WM-TI-664, Rev. 0

\subsubsection{Photoacoustic Spectroscopy}

In FTIR-Photoacoustic Spectroscopy (FTIR-PAS), the modulated infrared radiation absorbed by a sample is first converted to heat. When the heat propagates to the sample's surface, and subsequently into the helium gas within the photoacoustic cell, it causes pressure variations and generates a photoacoustic signal. When the sample's thickness is larger than the sample's thermal diffusion length, only the heat generated within the first thermal diffusion length from the sample's surface can propagate to the surface and generate acoustic signals (Rosencweig, 1980). Since the thermal diffusion length for most polymeric materials in the MIR region is in the range of a few to a few tenths of a micron, FTIR-PAS can be used as a near-surface analytical technique.

The FTIR-PAS method used at Hanford was developed by Ames Laboratory, Iowa State University (Ames, Iowa) for the quantitative analyses of highly radioactive waste sludges. Development of this method was funded by EM-50, Department of Energy (Washington, D. C.) and is in the process of being transferred to Westinghouse Hanford Company (WHC) for tank waste chemical characterization.

To facilitate this technology transfer, WHC has purchased identical/similar instrumentation (FTIR spectrometer and photoacoustic cell) used by Ames Laboratory. The FTIR system is a research-grade FTIR (Bio-Rad FTS 60A) spectrometer that is capable of sample examination from the far-infrared to the near-infrared region with extended capability to analyze materials in the visible and Ultraviolet. (VS/UV) regions by using appropriate light sources and beamsplitters. Minimal sample preparation (mounting sample on sample holder and freeze-drying at $77^{\circ} \mathrm{K}$ ) is needed.

The increasingly complex and heterogeneous nature of Hanford tank waste has intensified the need to probe the compositional variation of waste materials with depth. Step-scan FTIR-PAS depth profiling of heterogeneous samples has attracted attention due to the nondestructive nature of photoacoustic spectroscopy and the uniform samp1ing depth across the entire spectrum a17owed by the step-scan method. Dr. John McClelland and co-workers at Ames Laboratory have developed a new approach to separate the signals from different layers using step-scan interferometry operated in a phase modulation mode.

According to theory (Dittmar et.a1, 1991) reducing the speed of the FTIR interferometer enables deeper probe of a sample. But in a non-phased modulated experiment, different infrared frequencies are modulated at different acoustic frequencies so that photoacoustic signals generated by low frequencies probe deeper into the sample than those of higher frequencies. Also, photoacoustic saturation in which weak absorption bands are enhanced compared to strong bands can result in broadening of strong bands that wil1 
WHC-SD-WM-TI-664, Rev。 0

complicate spectral analysis.

Phase modulation has the desirable effect of modulating all wavelengths at the same Fourier frequency. Signals generated at the surface of the sample will reach the microphone of the photoacoustic cell faster than those from deeper regions of the sample. This will result in depth profiling of a region in a sample. Typical sampling depths range from a few microns to 100 microns. The limiting factor in depth profiling is the thermal diffusion length of the sample. Data manipulation can be used to determine the ordering of sample layers, to isolate the spectra of individual layers within a sample, and to determine the thickness of layers. These and other ways of extracting depth information with photoacoustic spectroscopy are being developed by Ames Laboratory for Westinghouse Hanford Company. 
WHC-SD-WM-TI-664, Rev. 0

\subsection{EXPERIMENTAL PROCEDURES}

\subsection{Description of Materials and Sample Preparation}

\subsubsection{Reference Materials}

Table 3-1 lists the reference materials used to examine the infrared absorption characteristics of individual components of the simulated waste tank waste materials. Except for disodium nickel ferrocyanide which was washed twice with distilled deionized water, centrifuged, and dried to constant weight at $120^{\circ} \mathrm{C}$, some of the reference materials were used without further treatment. When available, spectrophotometric grade chemicals were used to collect reference spectra.

\subsubsection{Waste Simulants}

Table 3-2 shows the waste simulants characterized in this study. Wet sludges with consistency similar to peanut butter were examined "as received" by fiber optics and modular transfer optics. For photoacoustic measurements, an aliquot of the wet simulated waste was smeared onto a specially fabricated sample holder, quickly frozen to prevent migration of soluble components of the waste to the sample surface, and allowed to dry in a freeze dryer maintained at $-50^{\circ} \mathrm{C}$ for 6 hours. Freeze-drying will change 1 iquid water to water crystals (ice) that sublimes under vacuum. This process will immobilize the soluble components rapidly, minimizing/eliminating segregation of the lighter components of the waste. Thus, surface composition will not be very different from that of the bulk material.

\subsection{Spectral Data Acquisition}

\subsubsection{Evanescent Field Absorbance Sensor (EFAS) Method}

The general layout of the evanescent field absorbance experimental setup for both NIR and MIR systems is illustrated in Figure 2-4. The beam emanating from the infrared source of the FTIR spectrometer was focused onto the inlet of the single-fiber probe using a fiber positioner. The light was propagated along the 1-m fiber passing through the trough containing the sludge (simulant). Using a fiber coupler, the unabsorbed light was transmitted direct7y to the MCT detector for the MIR system or to the InSb detector via a $0.5-\mathrm{m}$ silica fiber extension for the NIR system.

The stainless steel sampling trough was filled with the sludge and the pertinent fiber was pushed through the sludge. To minimize moisture evaporation and to overcome surface tension effects which tend to 7 ift the fiber out of the sludge, the trough was provided with a stainless steel cover. The transmitted power through the optical fiber was measured by the detector and the collected data were imported to a data reduction and analysis system. 
Table 3-1: INFRARED REFERENCE MATERIALS

\section{REFERENCE MATERIAL}

Sodium Nitrate

Sodium Nitrite

Sodium sulfate

Ferrous Ammonium Sulfate

Ammonium Sulfate

Potassium Sulfate

Potassium Phosphate

Sodium Monohydrogen Phosphate

Disodium Nickel Ferrocyanide

Sodium Ferrocyanide

Potassium Ferrocyanide

Nickel Ferrocyanide

Magnesium Ferrocyanide

Zinc Ferrocyanide

Sulfamic Acid

\section{CHEMICAL FORMULA}

$\mathrm{NaNO}_{3}$

$\mathrm{NaNO}_{2}$

$\mathrm{Na}_{2} \mathrm{SO}_{4}$

$\mathrm{FeSO}_{4} \cdot\left(\mathrm{NH}_{4}\right)_{2} \mathrm{SO}_{4}$

$\left(\mathrm{NH}_{4}\right)_{2} \mathrm{SO}_{4}$

$\mathrm{K}_{2} \mathrm{SO}_{4}$

$\mathrm{K}_{3} \mathrm{PO}_{4} \cdot \mathrm{XH}_{2} \mathrm{O}$

$\mathrm{Na}_{2} \mathrm{HPO}_{4} \cdot 12 \mathrm{H}_{2} \mathrm{O}$

$\mathrm{Na}_{2} \mathrm{NiFe}(\mathrm{CN})_{6} \cdot 3 \mathrm{H}_{2} \mathrm{O}$

$\mathrm{Na}_{4} \mathrm{Fe}(\mathrm{CN})_{6} \cdot 10 \mathrm{H}_{2} \mathrm{O}$

$\mathrm{K}_{4} \mathrm{Fe}(\mathrm{CN})_{6} \cdot 3 \mathrm{H}_{2} \mathrm{O}$

$\left.\mathrm{Ni}_{2} \mathrm{Fe}(\mathrm{CN})_{6} \cdot \mathrm{XH}_{2}\right)$

$\mathrm{Mg}_{2} \mathrm{Fe}(\mathrm{CN})_{6} \cdot 12 \mathrm{H}_{2} \mathrm{O}$

$\mathrm{Zn}_{2} \mathrm{Fe}(\mathrm{CN})_{6} \cdot 3 \mathrm{H}_{2} \mathrm{O}$

$\mathrm{NH}_{2} \mathrm{SO}_{3} \mathrm{H}$ 
WHC-SD-WM-TI-664, Rev. 0

Table 3-2: SIMULATED TANK WASTES

\author{
BY-104 Saltcake \\ In Farm 1 , Rev 28 and $29 \mathrm{~A}$ \\ In Farm 2, Rev 26A \\ $U$ Plant 1A-7A, Centrifuged Solids \\ $U$ Plant 2-5, Top Layer, Centrifuged Solids \\ U Plant 2-6, Bottom Layer, Centrifuged Solids \\ U Plant 2-9A, Top Fraction, Centrifuged Solids \\ $U$ Plant 2-19A, Bottom Fraction, Centrifuged Solids \\ SY-101, Window E with EDTA \\ T Plant "Special 1", Top Fraction \\ T Plant "Special 2", Bottom Fraction \\ T Plant 19A
}


WHC-SD-WM-TI-664, Rev. 0

Single beam spectra of the fibers were measured in air for background. Evanescent field spectra of the sludges were collected using 100 scans at a nominal resolution of $8 \mathrm{~cm}^{-1}$.

\subsubsection{Diffuse/Specular Reflectance Spectra Collection}

The NIR bifurcated bundle fiber optic probe described in Section 2.3.1.2 was connected to the fiber optic accessory shown in Figure 2-2 and then optimized for maximum signal (infrared source- tungsten filament, beamsplitter-quartz). The sample (wet/dry sludge) was placed in a small stainless steel sample cup located in a laboratory hood. Large particles of dry samples (BY-104) were reduced to powder $(<100 \mu \mathrm{m})$ using an agate mortar prior to sampling. The top surface of the sample in the sample cup was leveled using a stainless steel spatula. The sample was then ready for spectral data acquisition.

The fiber probe was introduced to the sample such that the probe window was just in contact with the sample (diffuse reflectance mode) or placed about $1 \mathrm{~mm}$ above the sample (specular reflectance mode). The sample was interrogated for its infrared-active components for a few seconds (32 scans at $16 \mathrm{~cm}^{-1}$ resolution). The infrared light from the source was diffused and partiy absorbed by the sample. The components of the light's returned light spectrum were then analyzed to determine the composition of the sample.

To obtain the background spectrum for the diffuse reflectance configuration, spectra-pure $\mathrm{NaCl}$, pulverized to similar sizes as those of the samples, was used. The spectrum of a flat aluminum mirror served as background spectrum for the specular reflectance configuration.

\subsubsection{Photoacoustic Spectra Measurement}

The instrumentation for photoacoustic (FTIR-PAS) spectra collection includes an FTIR spectrometer with step-scan capability (Bio-Rad FTS 60A) and a photoacoustic cell (MTec 200 ce11). The spectral range of the detector of the photoacoustic cell depends only on the transparency of the sample chamber window (McClelland, et a1. 1992). With suitable window, a detector can operate from the ultraviolet (UV) to the far-infrared (FIR). The most common window material is $\mathrm{KBr}$ (UV through MIR).

The freeze-dried sample (Section 3.1.2) or reference material was introduced into the photoacoustic cell and flushed with helium. Its photoacoustic spectrum was collected by co-addition of 32 scans at a resolution of $8 \mathrm{~cm}^{-1}$. This spectrum was then ratioed to that of carbon black used as background/reference. Ratioed spectra were collected in the MIR $\left(4000 \mathrm{~cm}^{-1}\right.$ to $500 \mathrm{~cm}^{-1}$ ) and NIR $\left(15,000 \mathrm{~cm}^{-1}\right.$ to $\left.4000 \mathrm{~cm}^{-1}\right)$ at scanning speeds of $2.5 \mathrm{kHz}$ and $800 \mathrm{~Hz}$, respective1y. 
WHC-SD-WM-TI-664, Rev。 0

\subsubsection{Modular Transfer Optics Using Light Guides}

Except for the demonstration of the capability of the system conducted by the vendor (Axiom Analytical, Irvine, CA), no work has been done on this method. In the demonstration, the vendor filled a small stainless steel sample cup with an aliquot of the wet sludge (In Farm ) and leveled the top surface with a stainless steel spatula. No further sample treatment was performed. The sample was examined using the modular transfer optic system interfaced to an FTIR. The infrared light from the FTIR source was conveyed to the samplers [diffuse reflectance (DR) and specular reflectance (SR) modes] located in a laboratory hood via the modular transfer optic system. The spectral data (DR and SR) showed great potential for quantifying water in waste sludges in the MIR region.

\subsubsection{Spectral Data Manipulation}

To obtain a spectrum that is characteristic of the sample, all effects and features of the FTIR-fiber probe system or FTIR-PAS system must be eliminated. This was done by first measuring a background spectrum. The background interferogram was Fourier transformed (FT) into a background single-beam spectrum. Similarly, the sample interferogram was collected, Fourier transformed into a sample single-beam spectrum, and then ratioed to the background single-beam spectrum to obtain a ratioed spectrum.

To produce a $\%$ transmittance spectrum $(\% T)$, the sample single-beam spectrum was divided by the background spectrum and displayed from 0 to $100 \%$. To produce an absorbance spectrum, the logarithm to the base 10 of the reciprocal of the transmittance spectrum was computed according to the equation, $A=\log _{10}(1 / T)$. The computed spectra were analyzed for chemical species of interest.

The NIR region is a quite complex area of the electromagnetic spectrum. There are a great many absorptions possible in this region due to overtones and combination bands. These absorptions are very close and cannot be individually resolved in most cases. There are also interfering factors such as baseline shifts and tails of absorptions from both the visible and MIR regions.

Derivatives of spectra have been used to study complex profiles with overlapping curves, or to study profiles where absorption maxima are superimposed on broad backgrounds. The derivative of a spectrum is the rate of change of the slope of the line describing the spectrum. An efficient way to calculate derivatives is to use Fourier domain calculations (Bio-Rad FTS 60A User's Manual). This involves two steps: application of the derivative weighting function and application of a truncating or smoothing function to the Fourier transform of the spectral region under study. The second step has the major effect as it permits the user to selectively eliminate the noise component and retain only the useful information. 
To obtain the derivative spectrum, the differentiation function of the software furnished by the FTIR vendor is used. The differentiation function generates an even-ordered derivative of the spectrum displayed in absorbance format. Generally, even-ordered derivatives are used to increase the resolution, so that small or subtle features can be identified. However, the signal-to-noise ratio rapidly decreases when high-order derivatives are used. Thus smoothing is often used prior to, or in conjunction with, the obtaining of derivatives.

The second derivative of a spectrum removes contributions of offset and slope in the original spectrum. When peaks are extremely broad and when overlap is severe, as in the NIR region, quantitation has been routinely accomplished by multivariate calibration methods which use second-derivative transformations as a preprocessing step. These calibrations. assume a linear relationship between intensities of second derivatives and peak heights. Comparison of intensities of bands in second-derivative spectra results in quantitation of species of interest. However, this condition holds only for spectra in which peak widths do not vary significantly. 
WHC-SD-WM-TI-664, Rev. 0

\subsection{RESULTS AND DISCUSSION}

\subsection{Fiber Optics Results}

\subsubsection{Evanescent Field Absorbance Sensor (EFAS)}

Figure 4-1 depicts a typical mid-infrared spectrum of a partially dried ferrocyanide-containing simulated waste (In Farm) obtained by fiber optics in the attenuated total reflectance-evanescent field absorbance (ATR/EFAS) mode. The two very sharp bands at $3616 \mathrm{~cm}^{-1}$ and $3549 \mathrm{~cm}^{-1}$ superimposed on a broad peak around $3400 \mathrm{~cm}^{-1}$ and the band at $1620 \mathrm{~cm}^{-1}$ are assigned to the $\mathrm{OH}$ stretching and $\mathrm{OH}$ bending modes, respectively, from the waters of crystallization of $\mathrm{Na}_{2} \mathrm{NiFe}(\mathrm{CN})_{6}$ in the waste. The broad band at $3400 \mathrm{~cm}^{-1}$ is due to the $\mathrm{OH}$ stretch of 1 iquid (free) water. The strong band at $2094 \mathrm{~cm}^{-1}$ is attributed to the $\mathrm{NiFe}(\mathrm{CN})_{6}{ }^{2-}$ moiety. The bands at $1788 \mathrm{~cm}^{-1}, 1363 \mathrm{~cm}^{-1}$, and $833 \mathrm{~cm}^{-1}$ are due to the nitrate $\left(\mathrm{NO}_{3}^{-}\right)$species. The other bands are not very wel1 delineated from the background so no definite band assignment is made on them.

Figure 4-2 compares the MIR spectrum of the dried sludge (In Farm) obtained by EFAS with those collected by a chalcogenide fiber bundle (MIR-ATR) and photoacoustic spectroscopy (MIR-PAS). The MIR-EFAS and MIR-PAS appear similar while the MIR-ATR spectrum shows a reduction in intensity of the ferrocyanide band at $2094 \mathrm{~cm}^{-1}$. This may be due to interference of the chalcogenide fibers of the fiber probe used to collect this spectrum that also absorbs in this region.

Figure 4-3 displays the spectra of pure water and wet sludge (In Farm) examined by modular transfer optics (Axiom Analytical) equipped with diffuse reflectance (DRS) and specular reflectance (SRS) samplers. The top spectrum displays the spectrum of distilled, deionized water obtained by specular reflection. The middle and bottom spectra are those of the partially dried sludge examined using SRS and DRS techniques, respectively. The SRS spectrum of the sludge seems identical to its DRS spectrum. As can be seen, very broad Tiquid water (free) bands appear at $3400 \mathrm{~cm}^{-1}$ (OH stretch) and $1650 \mathrm{~cm}^{-1}$ (bending mode of $\mathrm{HOH}$ ). These spectra are presented in absorbance format because it has been reported to be more appropriate for highly absorbing species than the Kubelka-Munk format commonly used for DR measurements.

Figure 4-4 presents typical spectra of the sludge in the near-infrared (NIR) region collected by a single-fiber silica probe (Rebagay et al. 1994). Except for fiber artifacts (i.e., bands due to the polyimide polymer cladding of the silica core), distinct bands are discerned at $7005 \mathrm{~cm}^{-1}, 6784 \mathrm{~cm}^{-1}$ and 5215 $\mathrm{cm}^{-1}$, in the partially dried sludge sample. The band at $5215 \mathrm{~cm}^{-1}$ is the combination band between the $\mathrm{OH}$ stretching and $\mathrm{OH}$ bending modes. The band at $6784 \mathrm{~cm}^{-1}$ is a combination band consisting of the combination band at $5215 \mathrm{~cm}^{-1}$ and the $\mathrm{OH}$ bending mode. Finally the grouping from $6800 \mathrm{~cm}^{-1}$ to $7150 \mathrm{~cm}^{-1}$ is mostly from the first overtone of the OH stretching vibrations. 


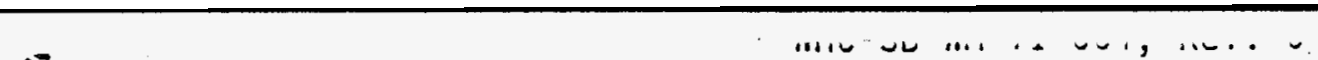

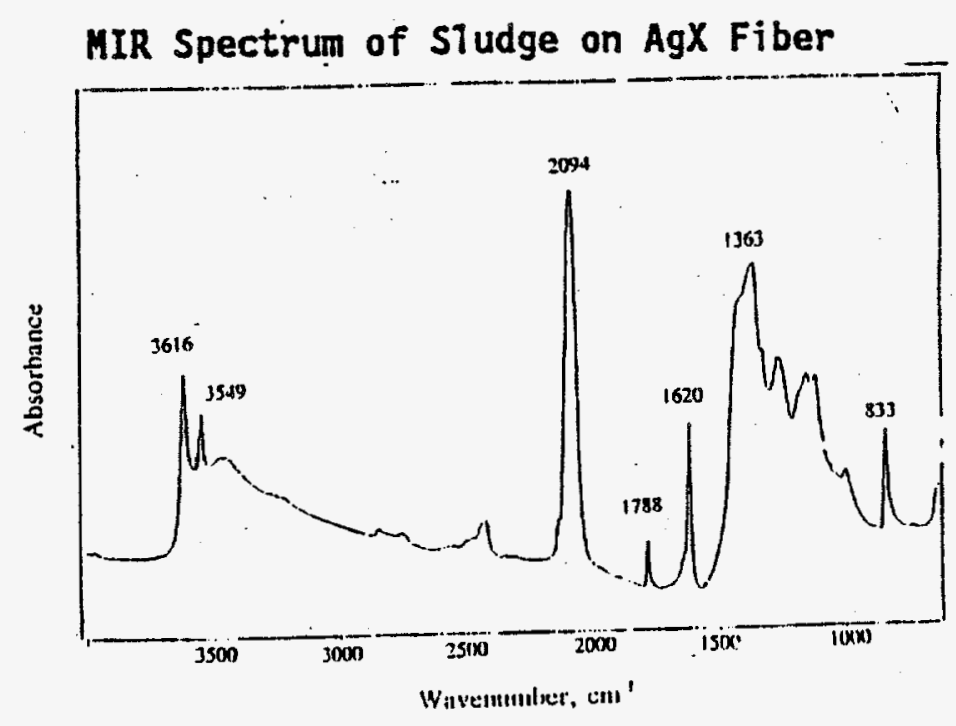

Figure 4-1. MIR Spectrum of STudge on AgX Fiber

Sludge $=$ In Farm AgX = Siver Bromide/Chloride

\section{Comparison of MIR Siudge Spectra}

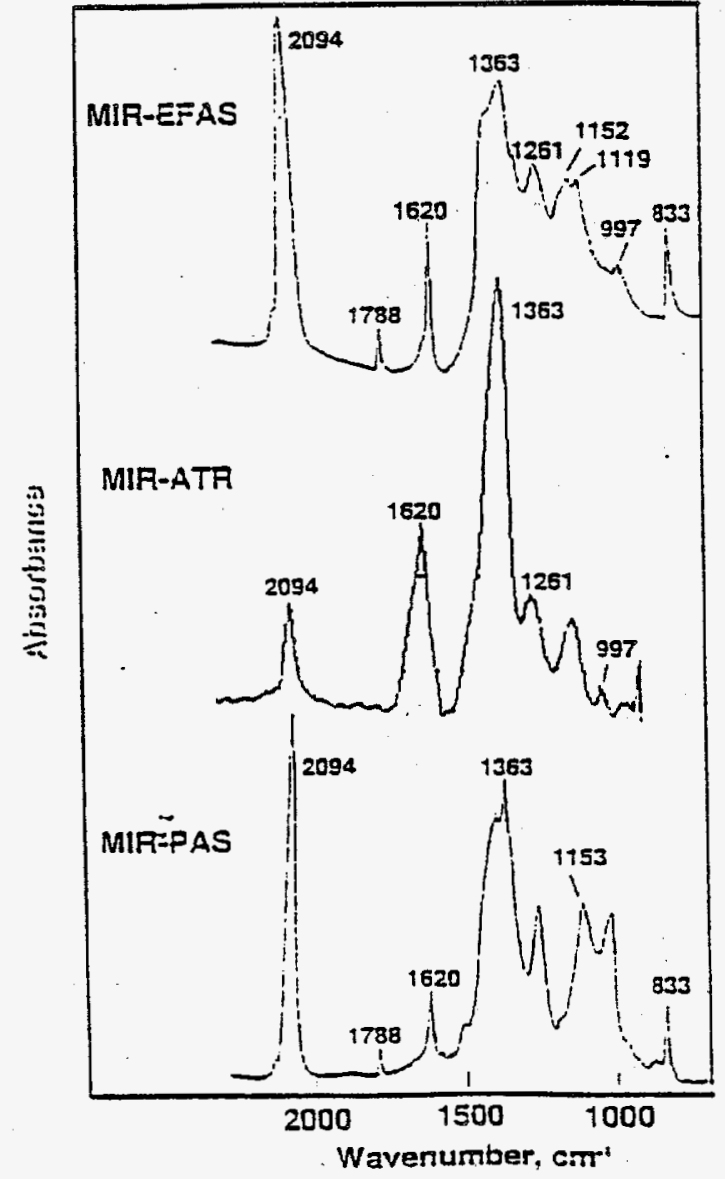

Figure 4-2. Comparison of MIR Sludge Spectra
Spectra of Siudge Using Light Pipes

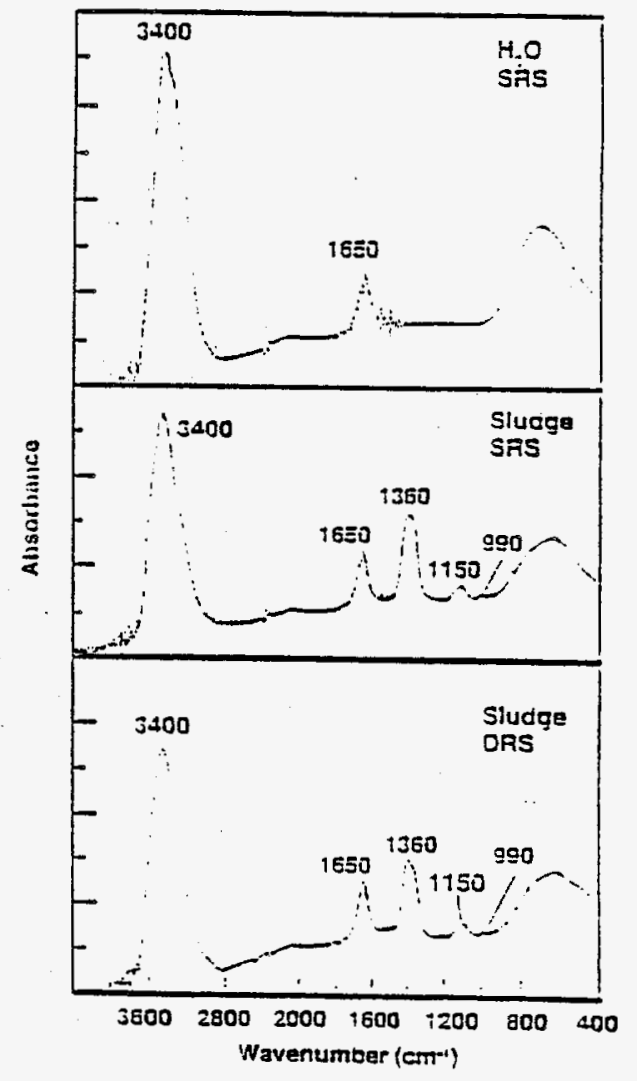

Figure 4-3. Spectra of Siudge Using Light Pipes 


\section{NIR Spectra of STudge on SiTica Fiber}

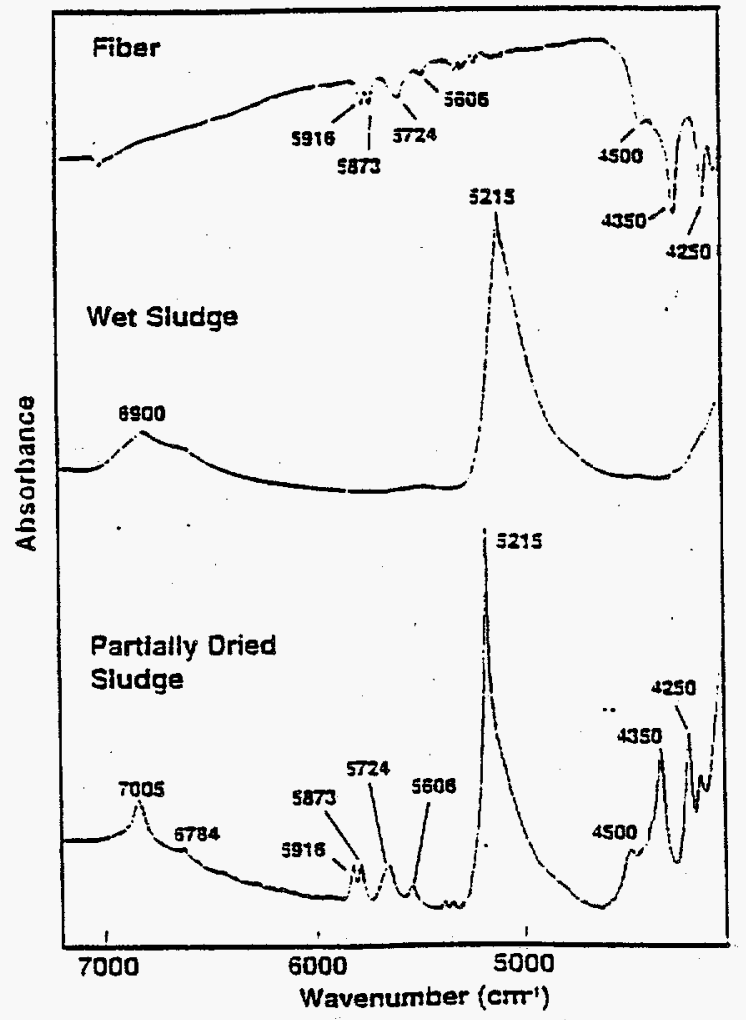

Disodium Nickel Ferrocyanide, $\mathrm{Na}_{2} \mathrm{NiFe}(\mathrm{CN})_{6}$

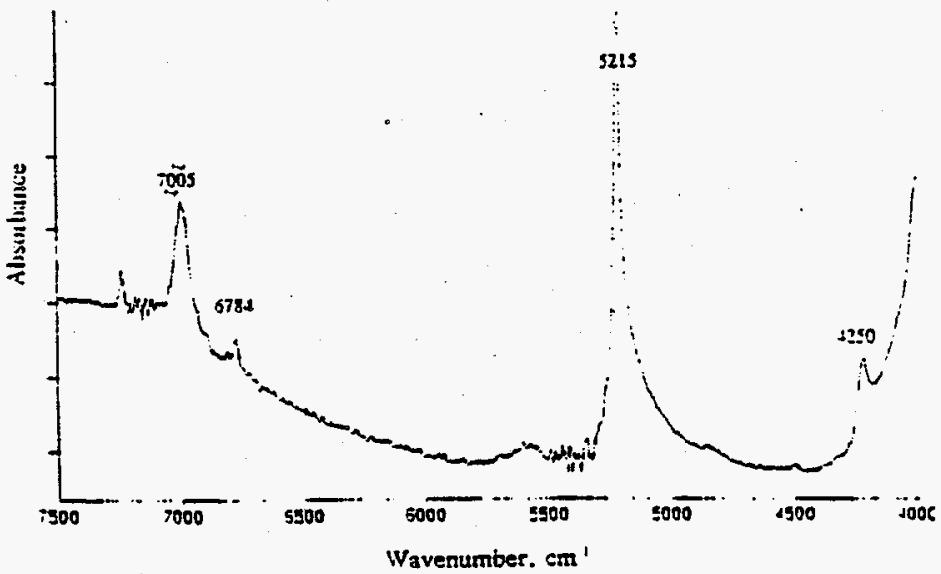

Figure 4-4. NIR Spectra of Sludge on Silica Fiber

Fiber Optic Probe $=$ Single Fiber EFAS
Figure 4-5. NIR Spectrum of Disodium Nickel Ferrocyanide Obtained by the SRL Fiber Probe 
WHC-SD-WM-TI-664, Rev. 0

\subsubsection{Diffuse Reflectance/Specular Reflectance Results}

Figure 4-5 depicts the NIR spectrum of $\mathrm{Na}_{2} \mathrm{NiFe}(\mathrm{CN})_{6}$ obtained by the bifurcated silica fiber optic probe made by Savannah River Laboratory (SRL). In addition to the water peaks located at $7005 \mathrm{~cm}^{-1}, 6784 \mathrm{~cm}^{-1}$, and $5215 \mathrm{~cm}^{-1}$, a sharp peak appears at $4250 \mathrm{~cm}^{-1}$. This peak is the first overtone of the $\mathrm{NiFe}(\mathrm{CN})_{6}$ species stretching mode $\left(2094 \mathrm{~cm}^{-1}\right)$. Because of fiber artifact interference in Figure 4-4, this peak can not be distinguished in the sludge. The appearance of the water peaks, however, indicate conclusively that the $\mathrm{Na}_{2} \mathrm{NiFe}(\mathrm{CN})_{6}$ is a hydrate.

Figure 4-6 shows NIR spectra of U Plant simulant using the Savannah River Laboratory (SRL) fiber optic probe. The top spectrum is that of the sample when the probe is touching the sample intimately (diffuse reflectance mode) while the spectrum at the bottom is when the probe is positioned at a distance of $1 \mathrm{~mm}$ above the sample (specular reflectance mode). It is interesting to note that there is no significant difference between these two configurations. Both spectra have similar features characterized by the presence of $\mathrm{OH}$ stretching first overtone and $\mathrm{OH}$ combination bands.

Figures 4-7 and 4-8 display the first and second derivative spectra of $U \mathrm{P} 7$ ant and SY-101 simulated sludges. It can be seen that subtle features of the original spectra can be identified after differentiation. The second derivative removes contribution of offset and slope in the original spectra.

Figures 4-9, 4-10, and 4-11 present the spectra of freeze-dried samples of In Farm, T Plant, and U PIant simulants obtained by the SRL fiber optic probe. A11 of the spectra indicate the presence of water bands at $7005 \mathrm{~cm}^{-1}$ and 5215 $\mathrm{cm}^{-1}$. The band at $7005 \mathrm{~cm}^{-1}$ of the T Plant simulant is weak and not very well defined. The broadening of the band at $5150 \mathrm{~cm}^{-1}$ may be indicative of the presence of more liquid/free water or other components of the simulant that absorb in this region.

\subsection{Photoacoustic Spectroscopy Results}

\subsubsection{Reference Spectra of Some Ferrocyanide Compounds}

Figure 4-12 to Figure 4-17 indicate the NIR and MIR photoacoustic (FTIR-PAS) spectra of some selected ferrocyanide compounds that may be used as reference.

All of these compounds are hydrates as shown by the water bands located at $3616,3549,1620,5215,6784$, and $7005 \mathrm{~cm}^{-1}$. In addition, a water 1 ibrational band appears at $740 \mathrm{~cm}^{-1}$. These bands are subject to shifts as a result of variations in hydrogen bonding with the ferrocyanide salt. It is apparent that more liquid water is involved in hydrogen bonding with the ferrocyanide in hydrates containing large amounts of water of crystallization. This is 
WHC-SD-WM-TI-664, Rev. 0

indicated by the broadening of the group of $\mathrm{OH}$ stretch bands near $3400 \mathrm{~cm}^{-1}$. In liquid water, a strong band is observed at $3400 \mathrm{~cm}^{-1}$ due to the symmetric and antisymmetric stretch of $0-\mathrm{H}$. A medium intensity band at $1648 \mathrm{~cm}^{-1}$ is due to the $\mathrm{H}-\mathrm{O}-\mathrm{H}$ bend. Liquid water al so produces a 7 ibrational band at $740 \mathrm{~cm}^{-1}$. The distinct ferrocyanide bands that can be seen unambiguously are the fundamental $\mathrm{CN}$ vibration at $2094 \mathrm{~cm}^{-1}$ and its first overtone at $4250 \mathrm{~cm}^{-1}$.

\subsubsection{Reference Spectra of Individual Simulant Components}

Figure 4-18 to Figure 4-27 are PAS spectra of individual components of the simulant wastes. All compounds were examined without pretreatment.

All of these spectra were collected during two technology transfer sessions conducted at Westinghouse Hanford Company's 222-S Laboratories and Ames Laboratory of Iowa State University. In general, all of these reference materials do not absorb or at most, absorb negligibly at the water absorption regions in the near-infrared region. Hence, using the NIR region for moisture determination in simulated and actual wastes is recommended.

\subsubsection{Photoacoustic (PAS) Spectral Data of Simulated Wastes}

Note: All simulated waste samples were freeze-dried prior to PAS examination.

The NIR and MIR spectra of BY-104 simulant are displayed in Figure 4-28. No ferrocyanide species are present as indicated by the absence of the fundamental stretching vibration of the cyanide band $\left(2094 \mathrm{~cm}^{-1}\right)$. Although a band appears at $4250 \mathrm{~cm}^{-1}$ in the NIR spectrum, this band may be due to the nitrate species that absorbs also at this location. The bands at $1788 \mathrm{~cm}^{-1}$, $1363 \mathrm{~cm}^{-1}, 833 \mathrm{~cm}^{-1}$ are due to nitrate species.

Figure 4-29 indicates the NIR and MIR spectra of In Farm simulated waste. The presence of ferrocyanide is indicated by the bands at $2094 \mathrm{~cm}^{-1}$ and $4250 \mathrm{~cm}^{-1}$ (not labeled). It would be worth investigating whether the ratio of the intensity of the water band at $5215 \mathrm{~cm}^{-1}$ to that of the ferrocyanide band at $4250 \mathrm{~cm}^{-1}$, after spectral differentiation preprocessing, can be used as a practical indicator for simultaneous screening of water and ferrocyanides.

Figure 4-30 shows the NIR and MIR spectra of SY=101 simulated waste. Since there is no fundamental stretching band for cyanides in the ferrocyanide region (MIR spectrum), it can be concluded that SY-101 does not contain ferrocyanide species.

Figure 4-31 displays the NIR and MIR spectra of T Plant simulated waste. The appearance of the ferrocyanide bands in both the MIR and NIR is conclusive evidence of the presence of ferrocyanides. Perhaps the intensity ratio, Intensity of $5215 \mathrm{~cm}^{-1}$ band/ Intensity of $3400 \mathrm{~cm}^{-9}$ band, can serve also as a monitor for free- and bound-water. 
WHC-SD-WM-TI-664, Rev 。 0

Figure 4-32 depicts the NIR and MIR spectra of $U$ Plant simulated waste. The ferrocyanide band can be discerned in the MIR region but the band at $4250 \mathrm{~cm}^{-1}$ in the NIR region is very weak (shoulder on a sloping background).

Application of the differentiation function (second derivative) may improve the resolution of the weak ferrocyanide peak.

Work will be pursued in the future to determine whether calculating the ratio of the intensity of the water band to that of the ferrocyanide band in the NIR region will result in the simultaneous measurement of water and ferrocyanide in actual Hanford Site wastes. 

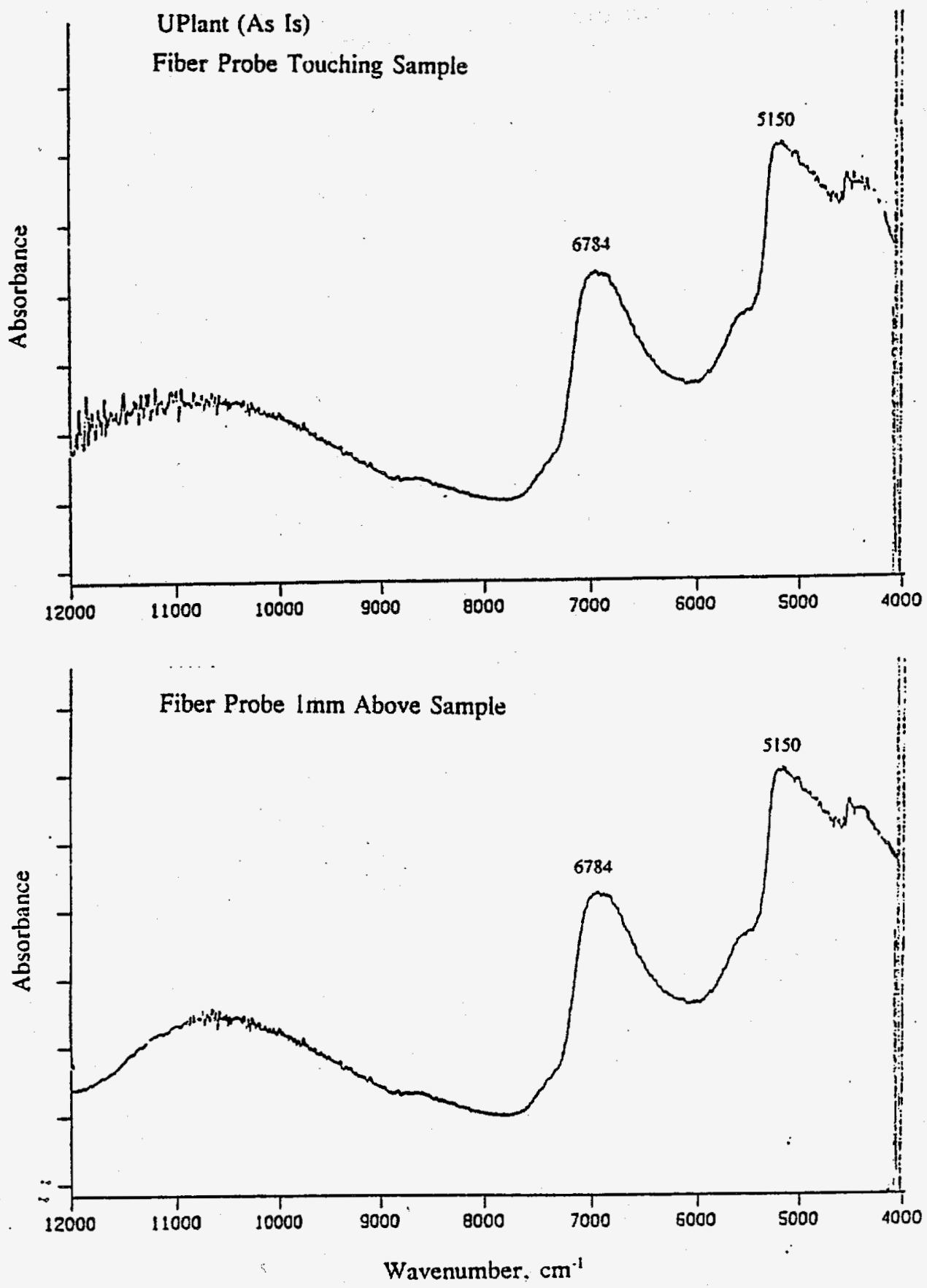

Figure 4-6. NIR Spectra of U Plant Simulant Using SRL Fiber Probe 
WHL-১U-WI'I-IL-004, KEV. U

UPlant (As Is)
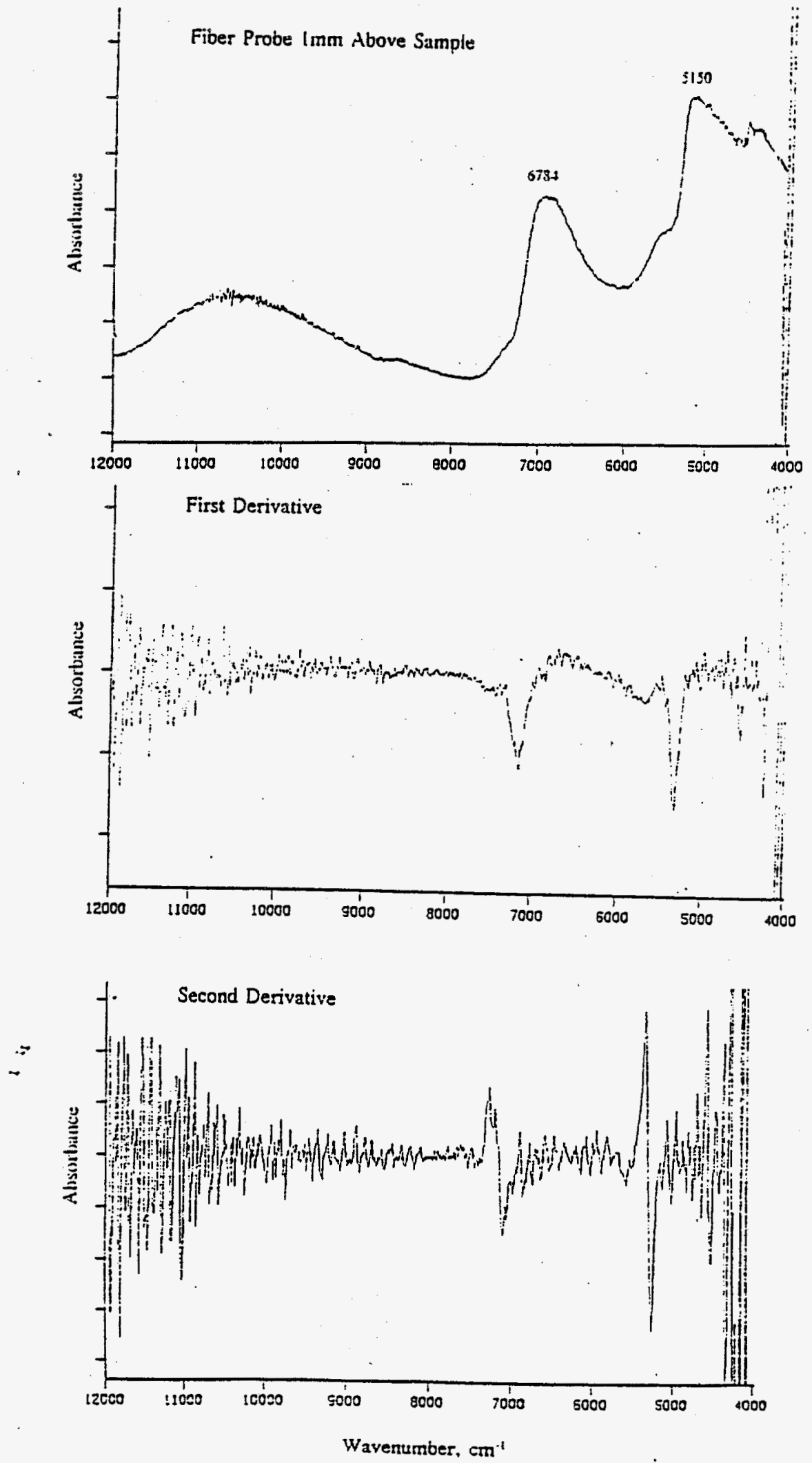

Figure 4-7. Derivative Spectra of U Plant Simulant 

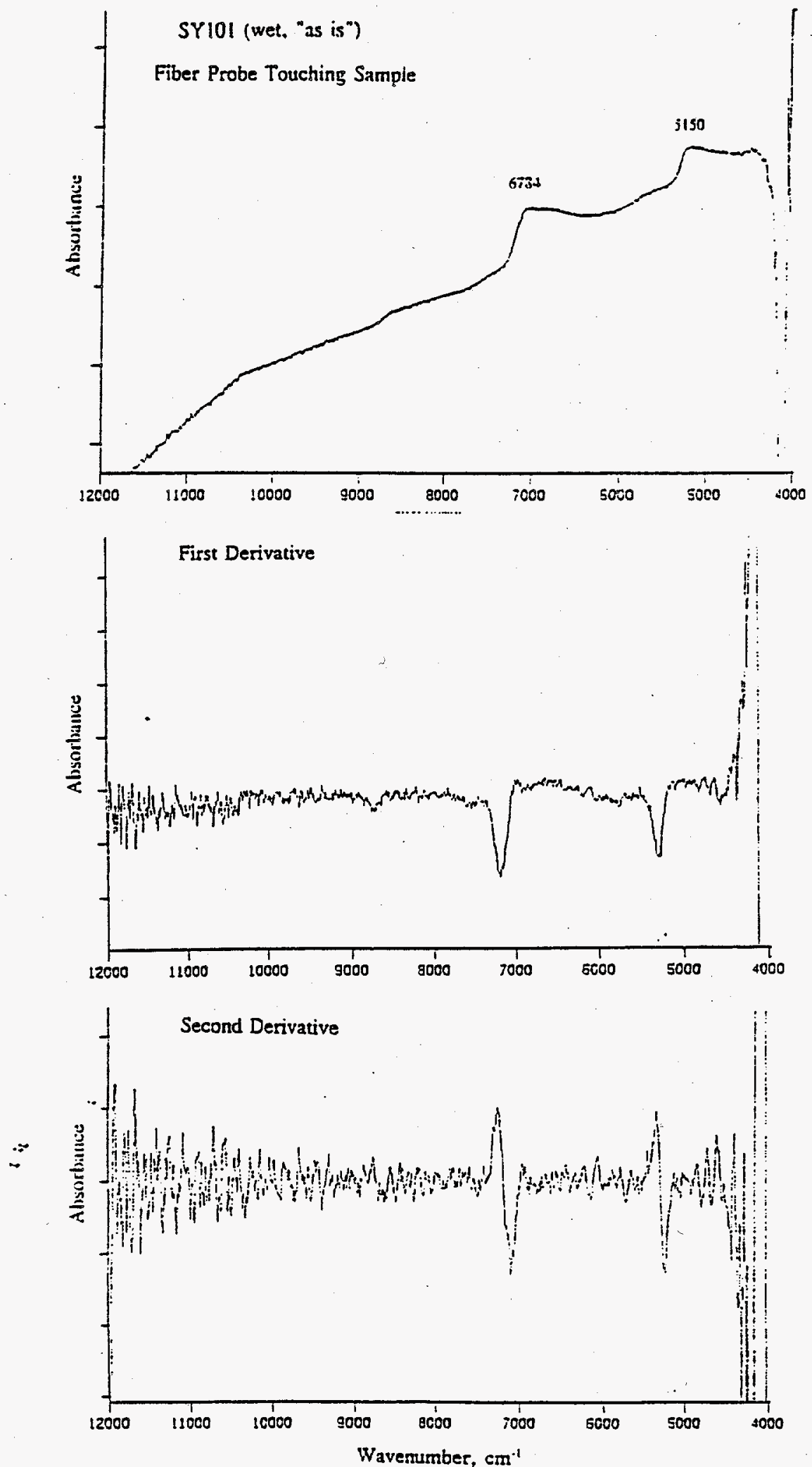

Figure 4-8. Derivative Spectra of SY-101 Simulant 

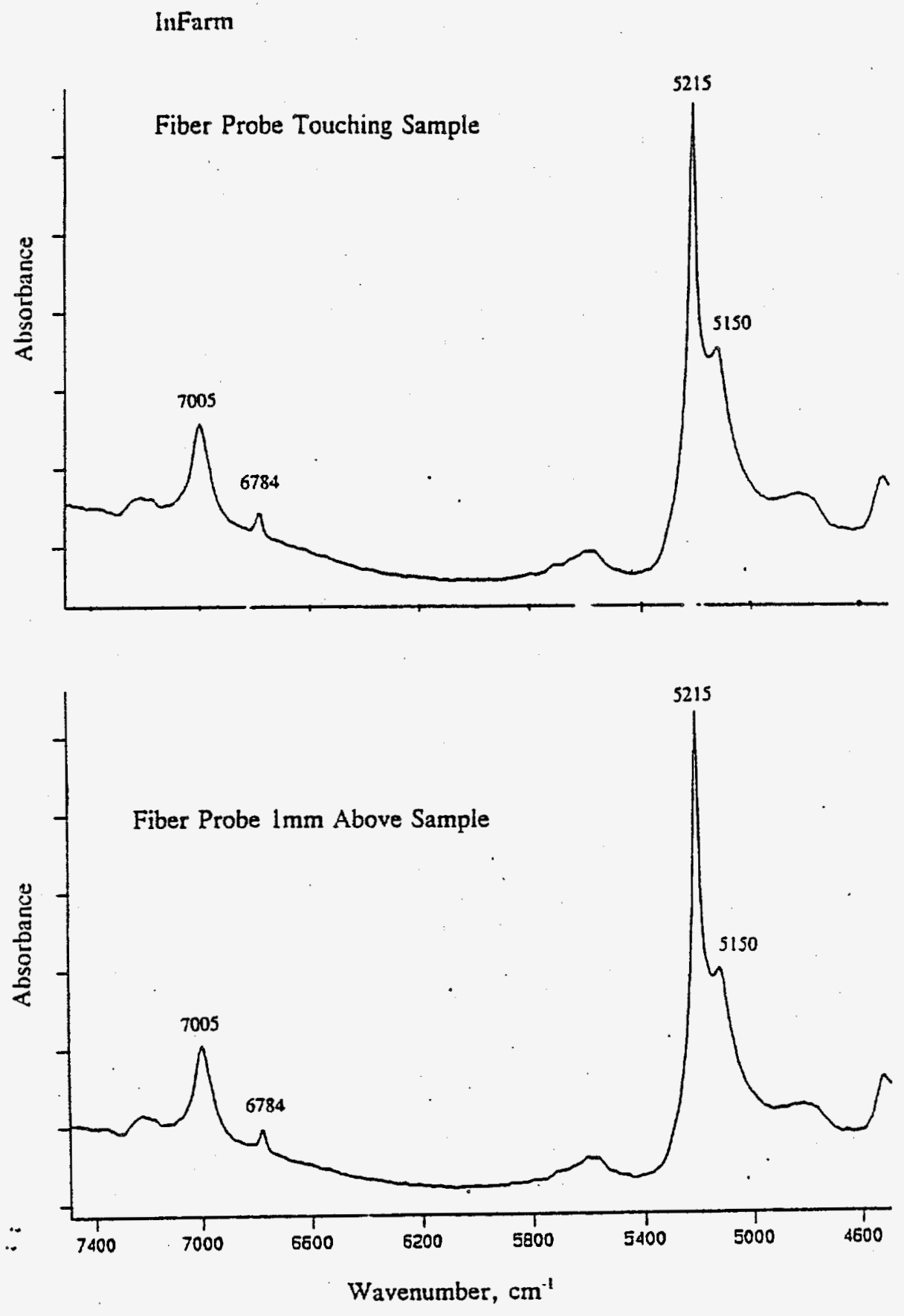

Figure 4-9. NIR Spectra of Dried In Farm Simulant

Fiber Probe $=$ SRL Fiber Optic Probe 

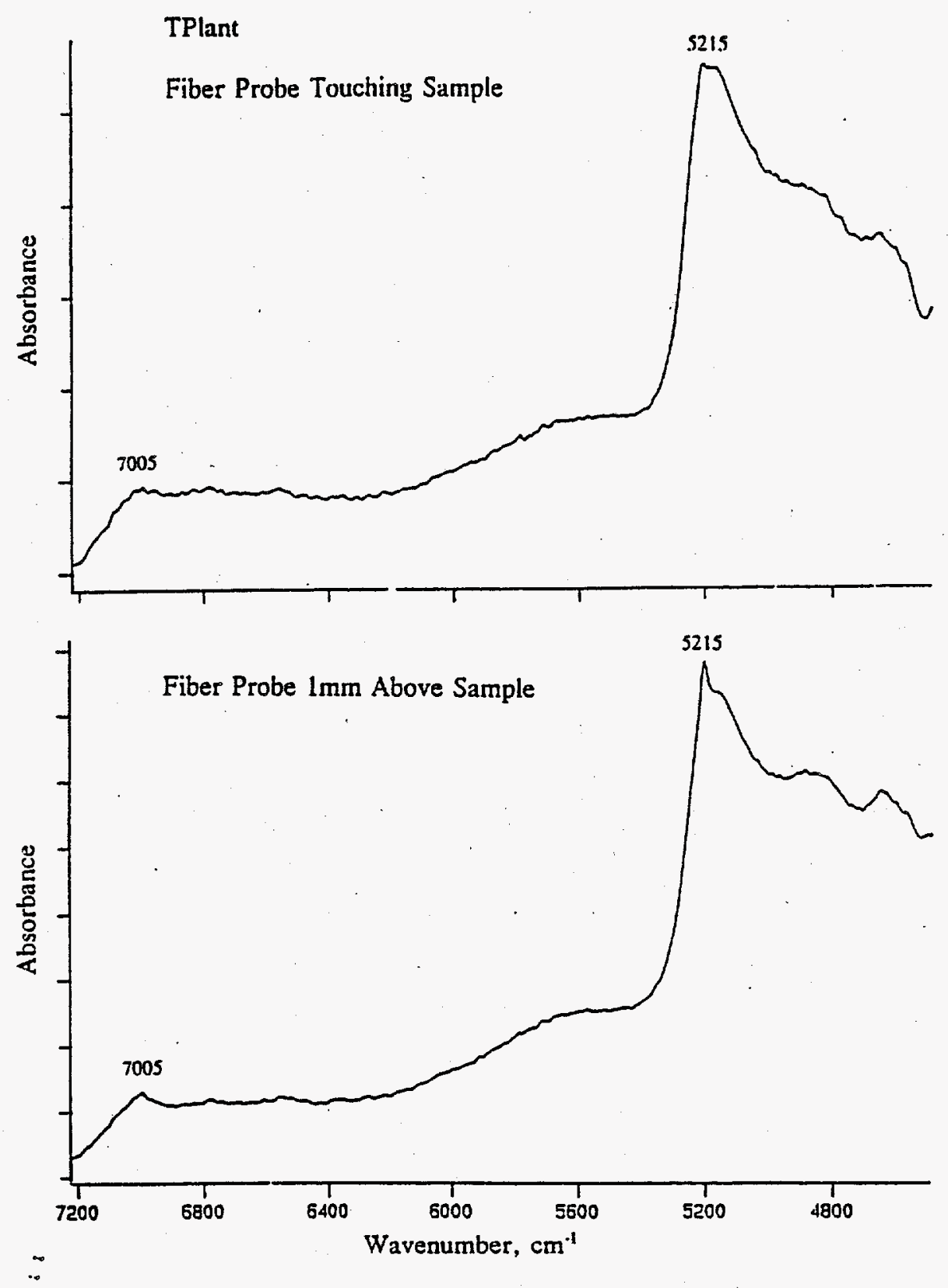

Figure 4-10. NIR Spectra of Oried T Plant Simulant

Fiber Probe $=$ SRL Fiber Optic Probe 


$\because \quad \quad \ldots \ldots<\ldots \ldots, \ldots, \ldots$
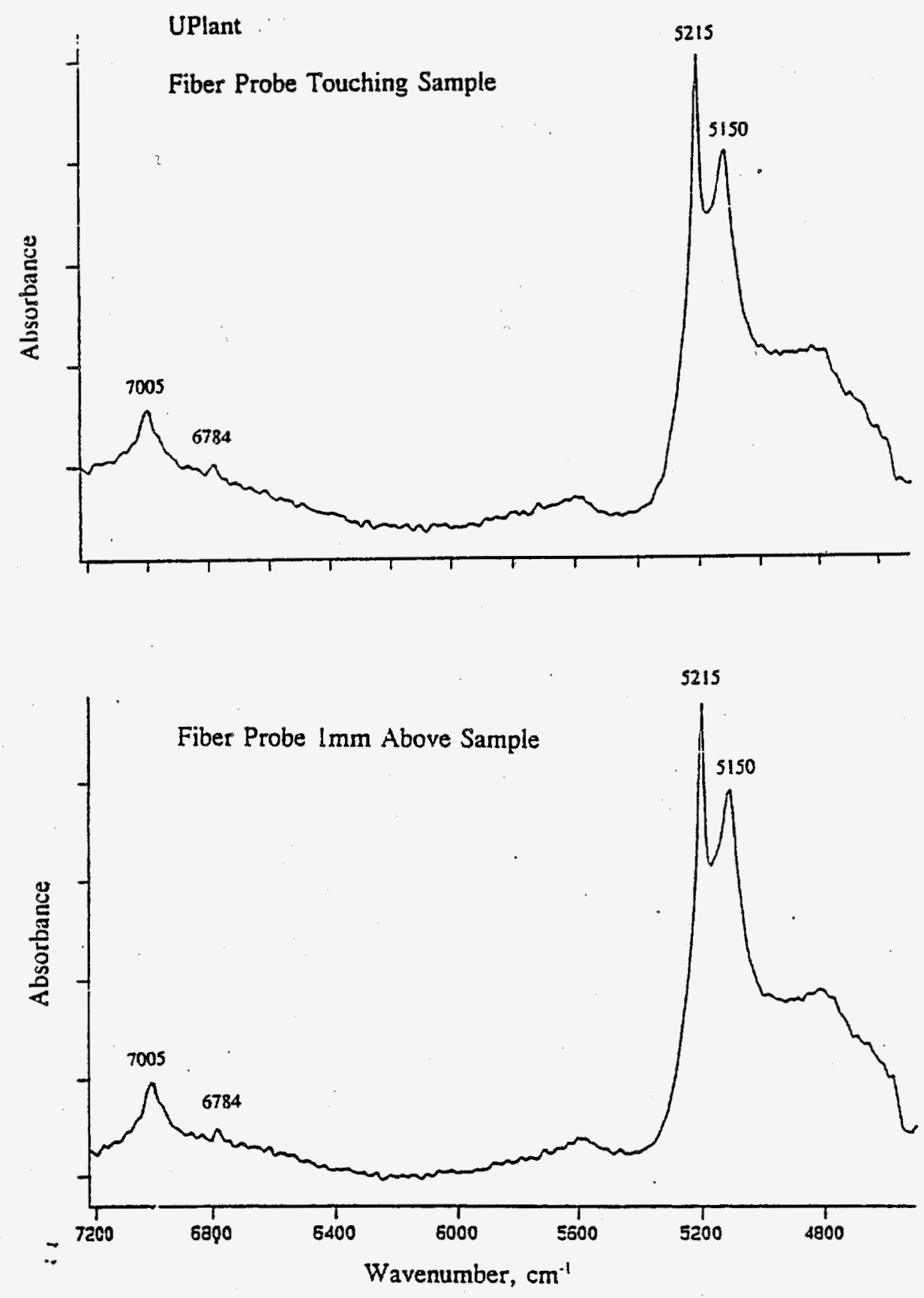

Figure 4-11. NIR Spectra of Dried U Plant Simulant

Fiber Probe $=$ SRL Fiber Optic Probe 
Disodium Nickel Ferrocyanide, $\mathrm{Na}_{2} \mathrm{NiFe}(\mathrm{CN})_{6}$
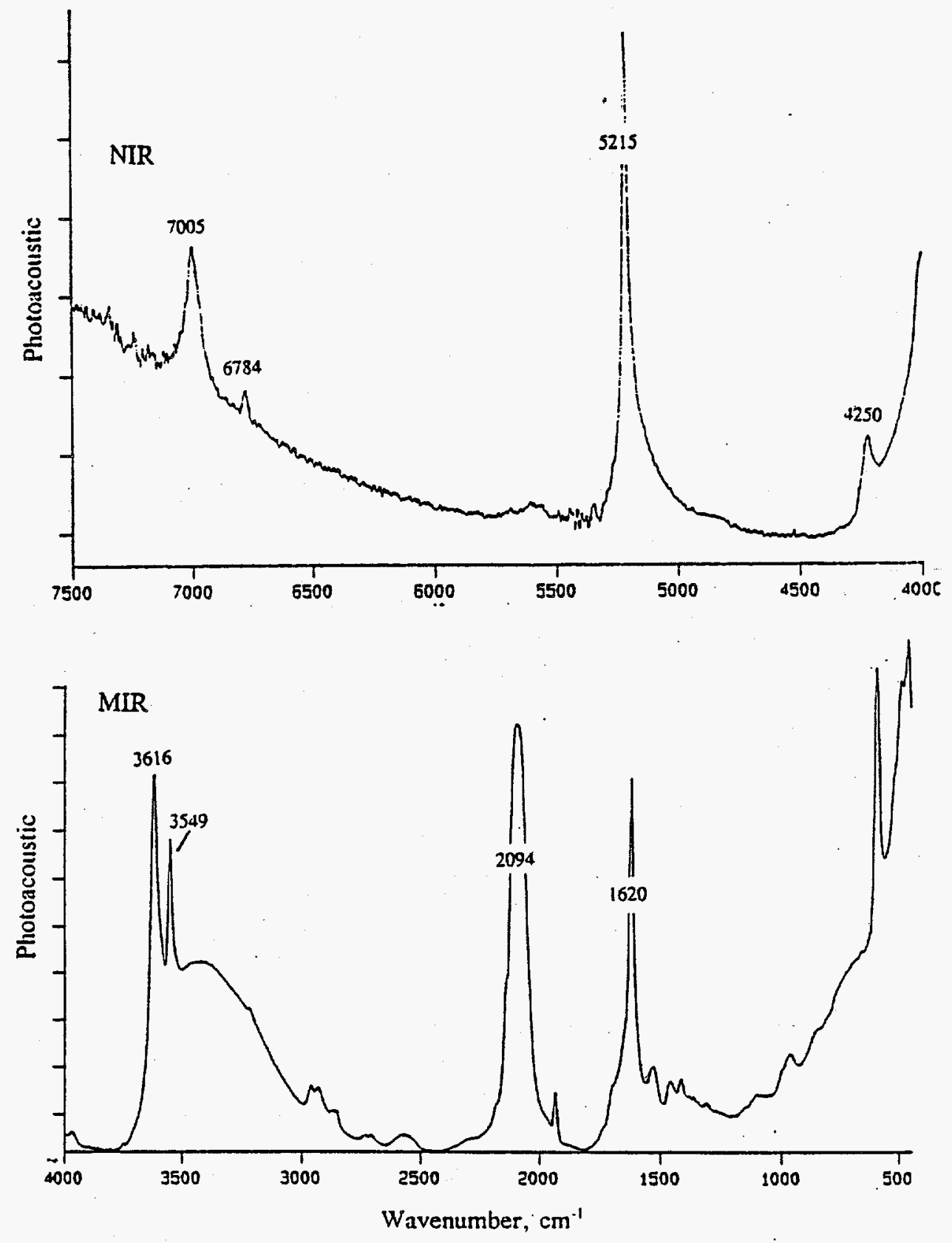

Figure 4-12. NIR and MIR Spectra of Disodium Nickel Ferrocyanide Note: Photoacoustic Spectroscopy 

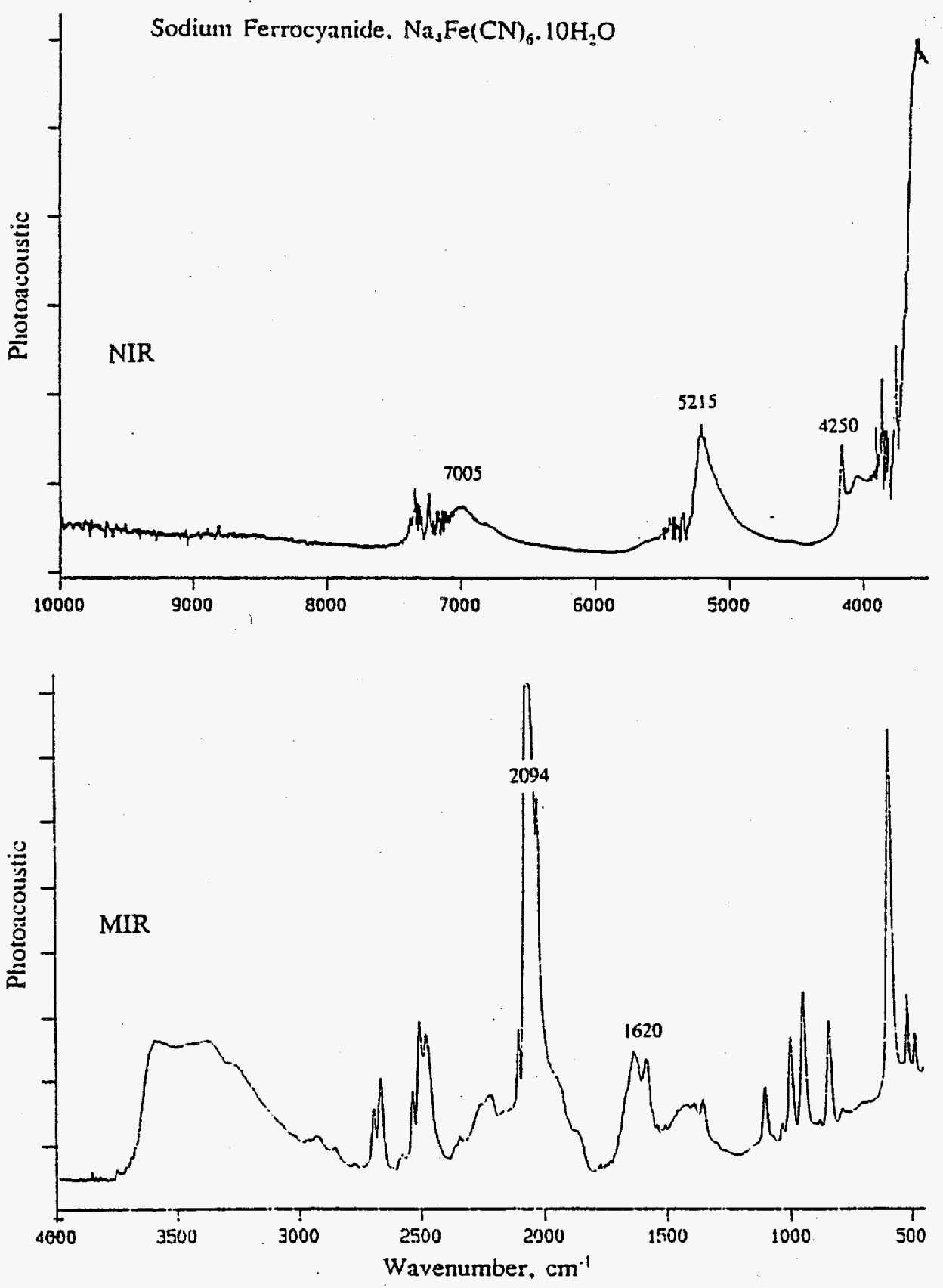

Figure 4-13. NIR and MIR Spectra of Sodium Ferrocyanide Note: Photoacoustic Spectroscopy 

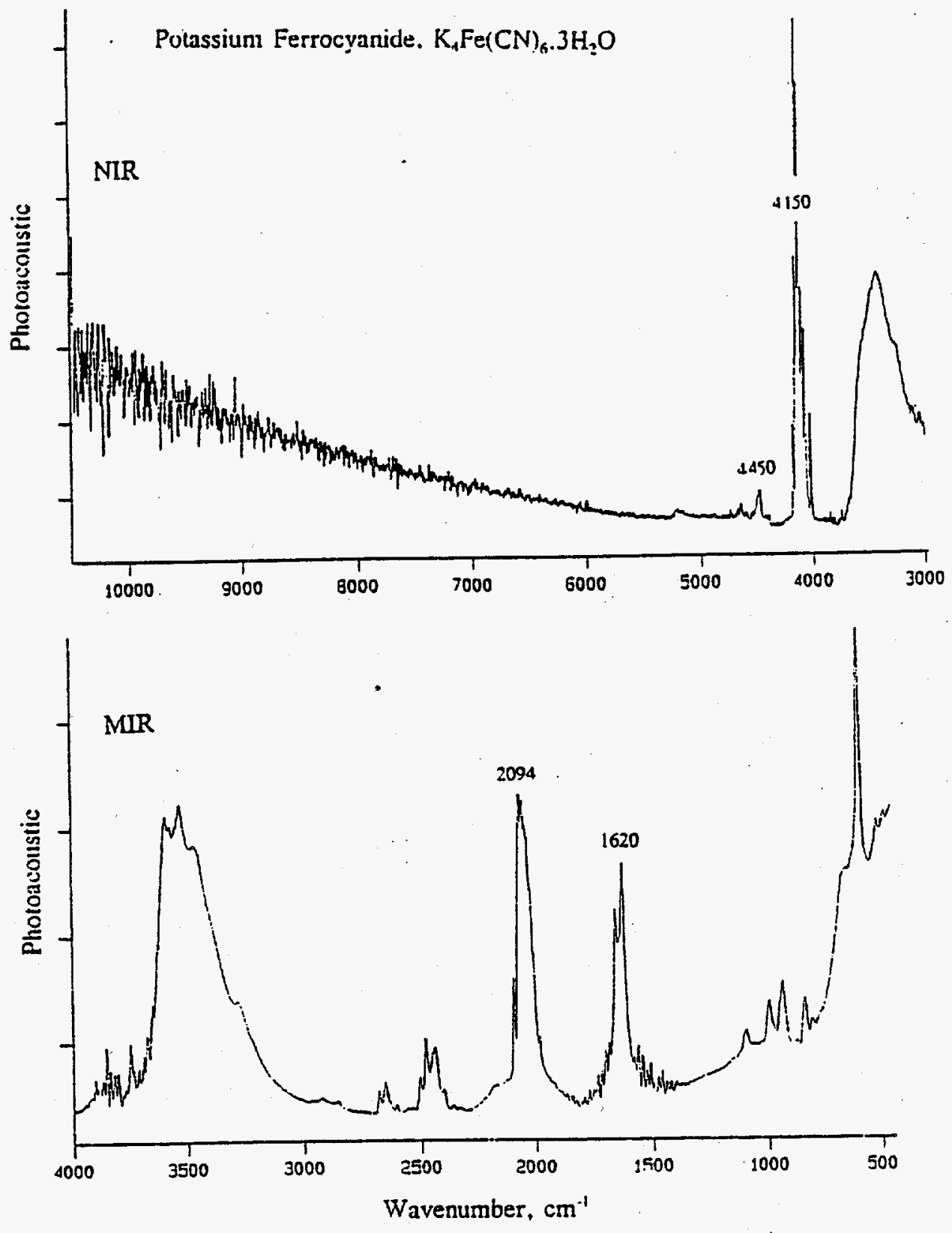

$=$

Figure 4-14. NIR and MIR Spectra of Potassium Ferrocyanide Note: Photoacoustic Spectroscopy 

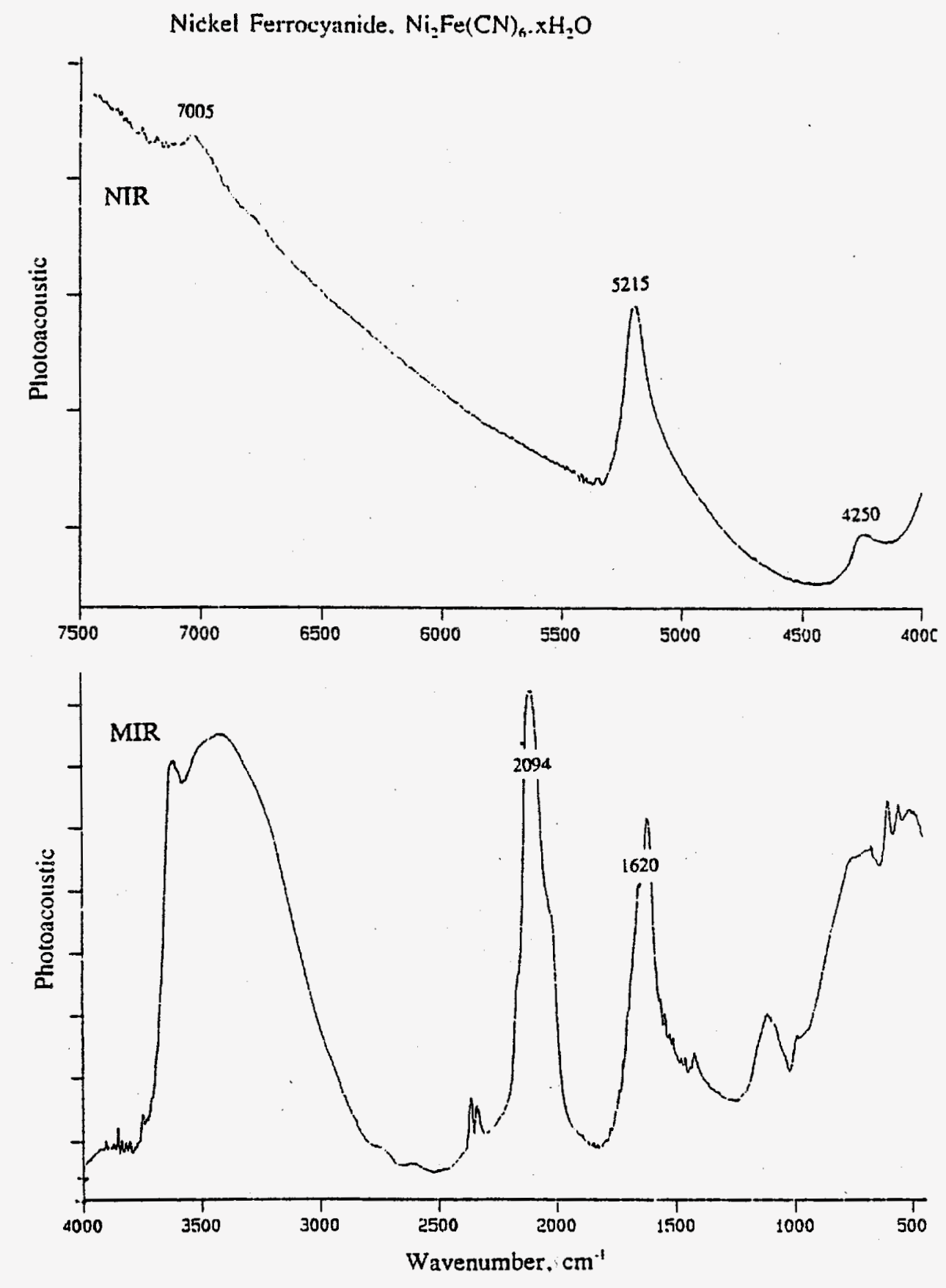

Figure 4-15. NIR and MIR Spectra of Nickel Ferrocyanide Note: Photoacoustic Spectroscopy 

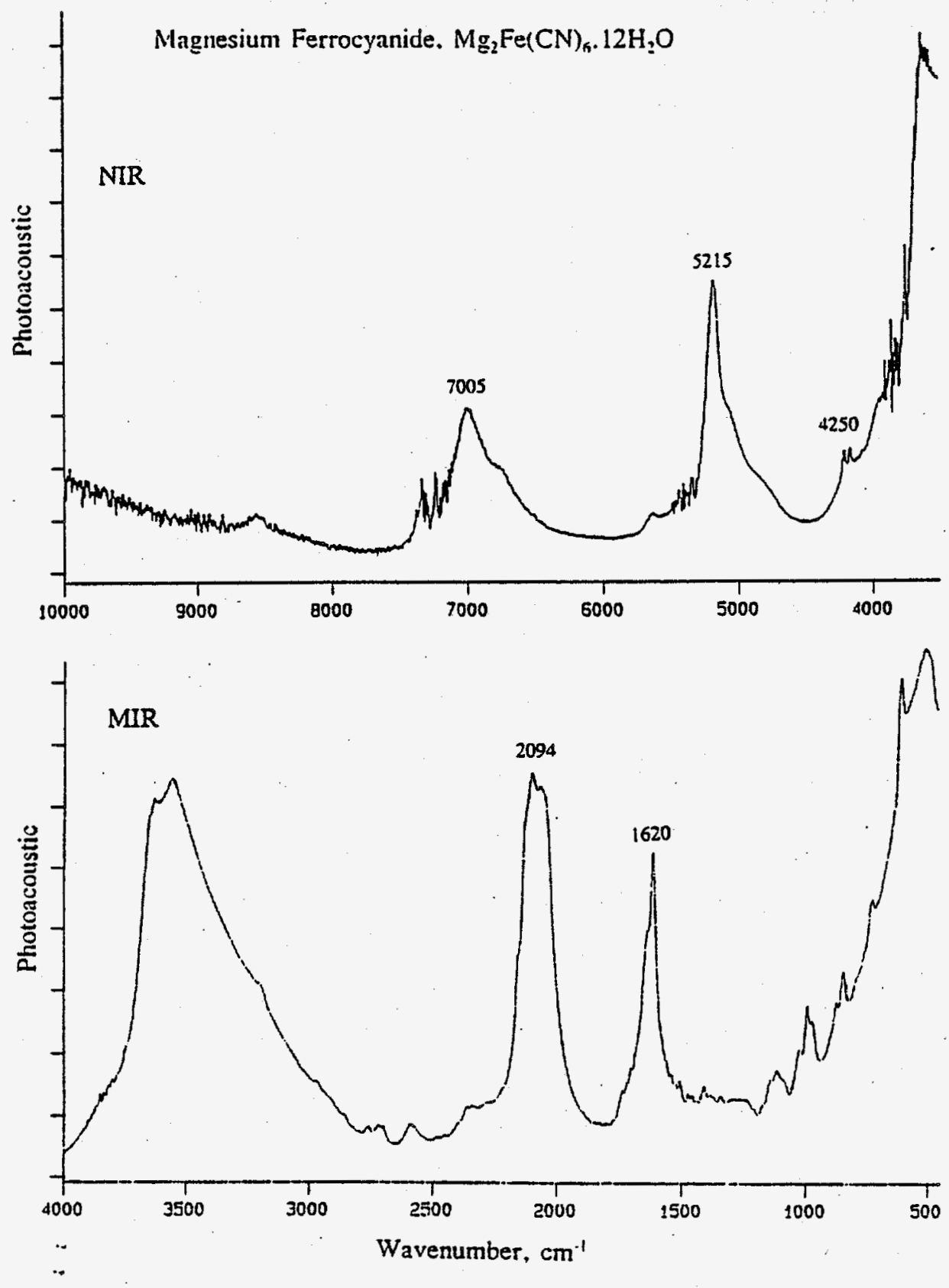

Figure 4-16. NIR and MIR Spectra of Magnesium Ferrocyanide Note: Photoacoustic Spectroscopy 

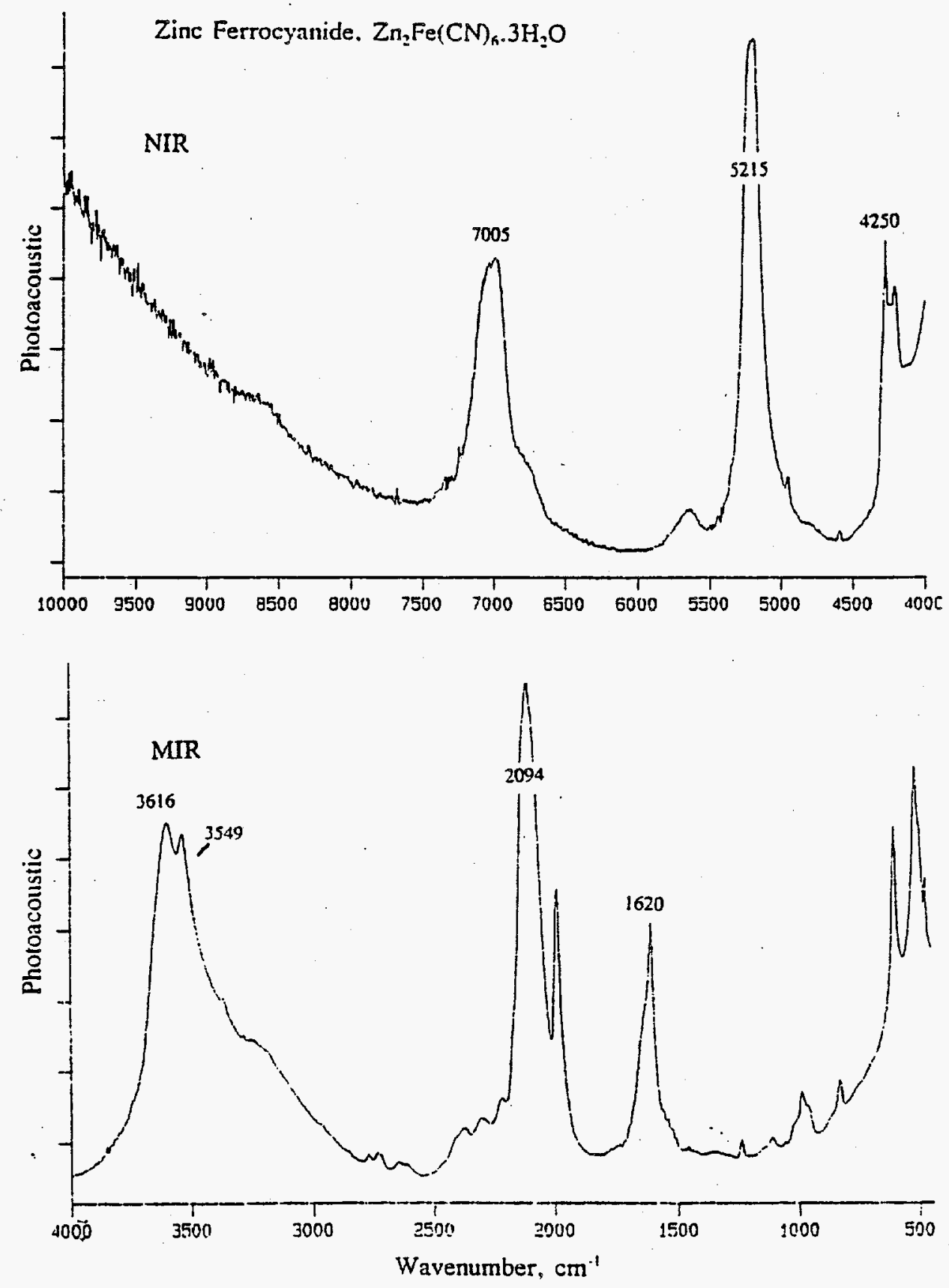

Figure 4-17. NIR and MIR Spectra of Zinc Ferrocyanide Note: Photoacoustic Spectroscopy 
WHC-SD-WM-11-664, Kev. U
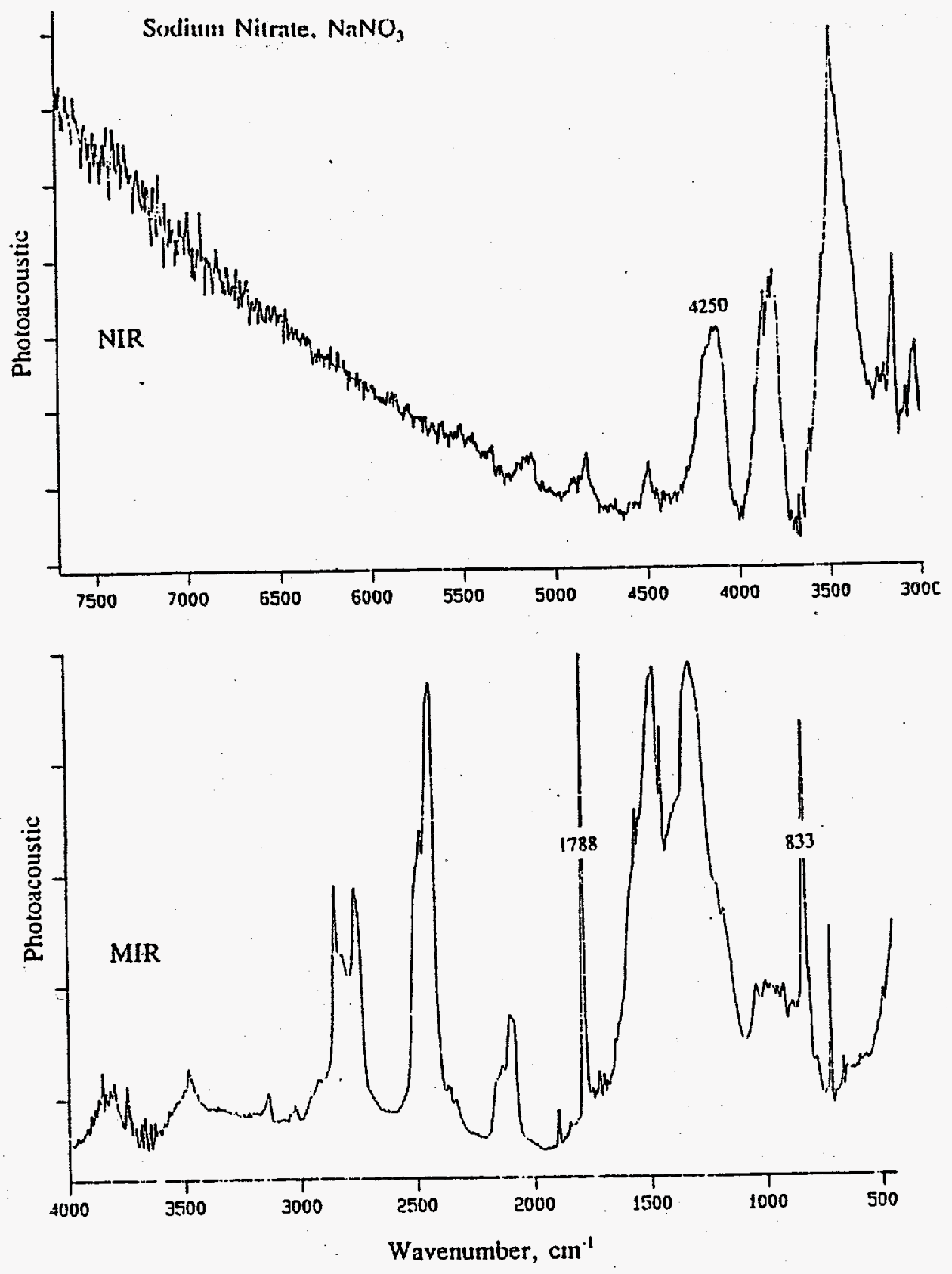

$\because$

Figure 4-18. NIR and MIR Spectra of Sodium Nitrate Note: Photoacoustic Spectroscopy 

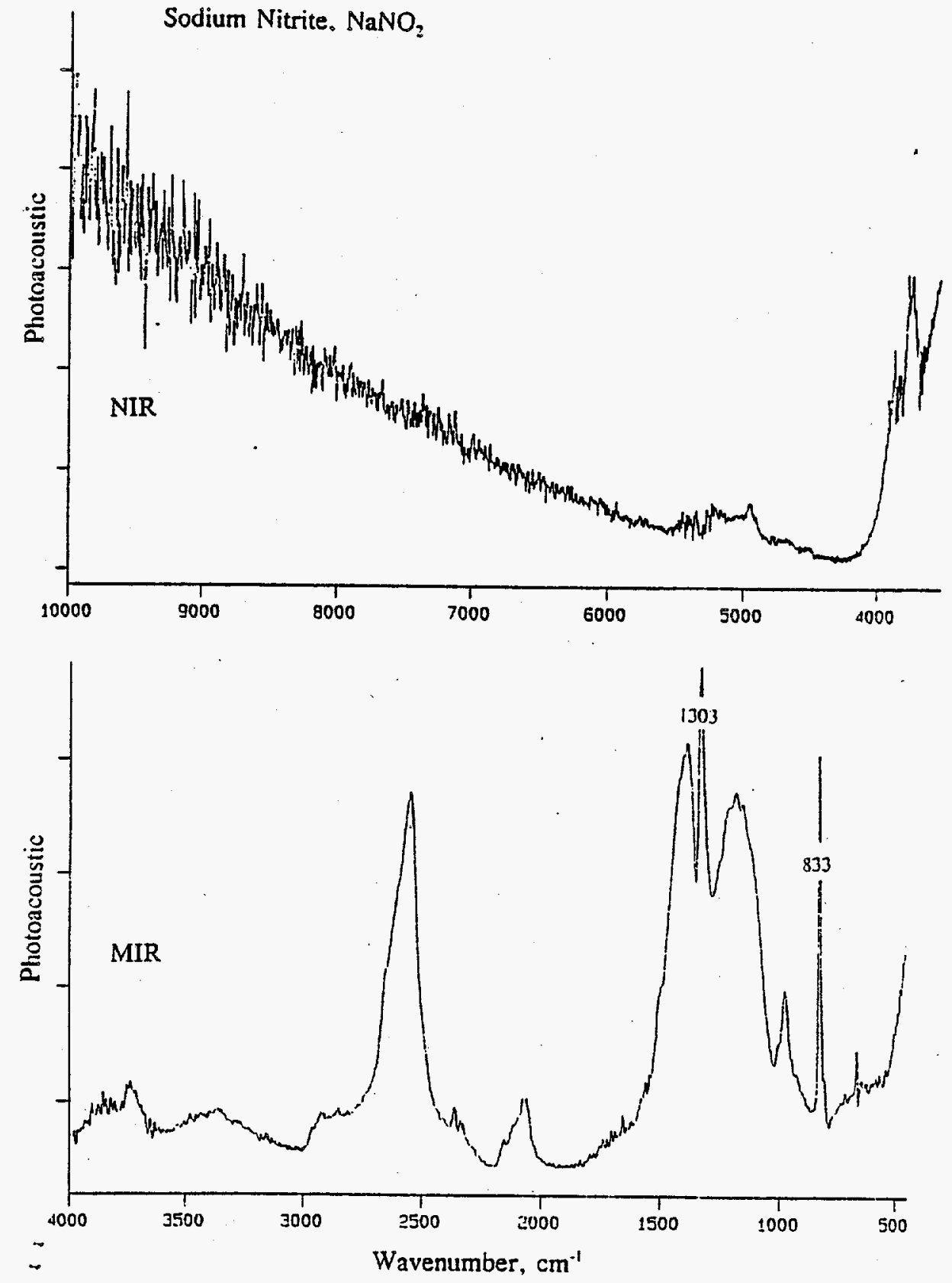

Figure 4-19. NIR and MIR Spectra of Sodium Nitrite Note: Photoacoustic Spectroscopy 
WHC-SD-WM-TI-664, Rev. 0
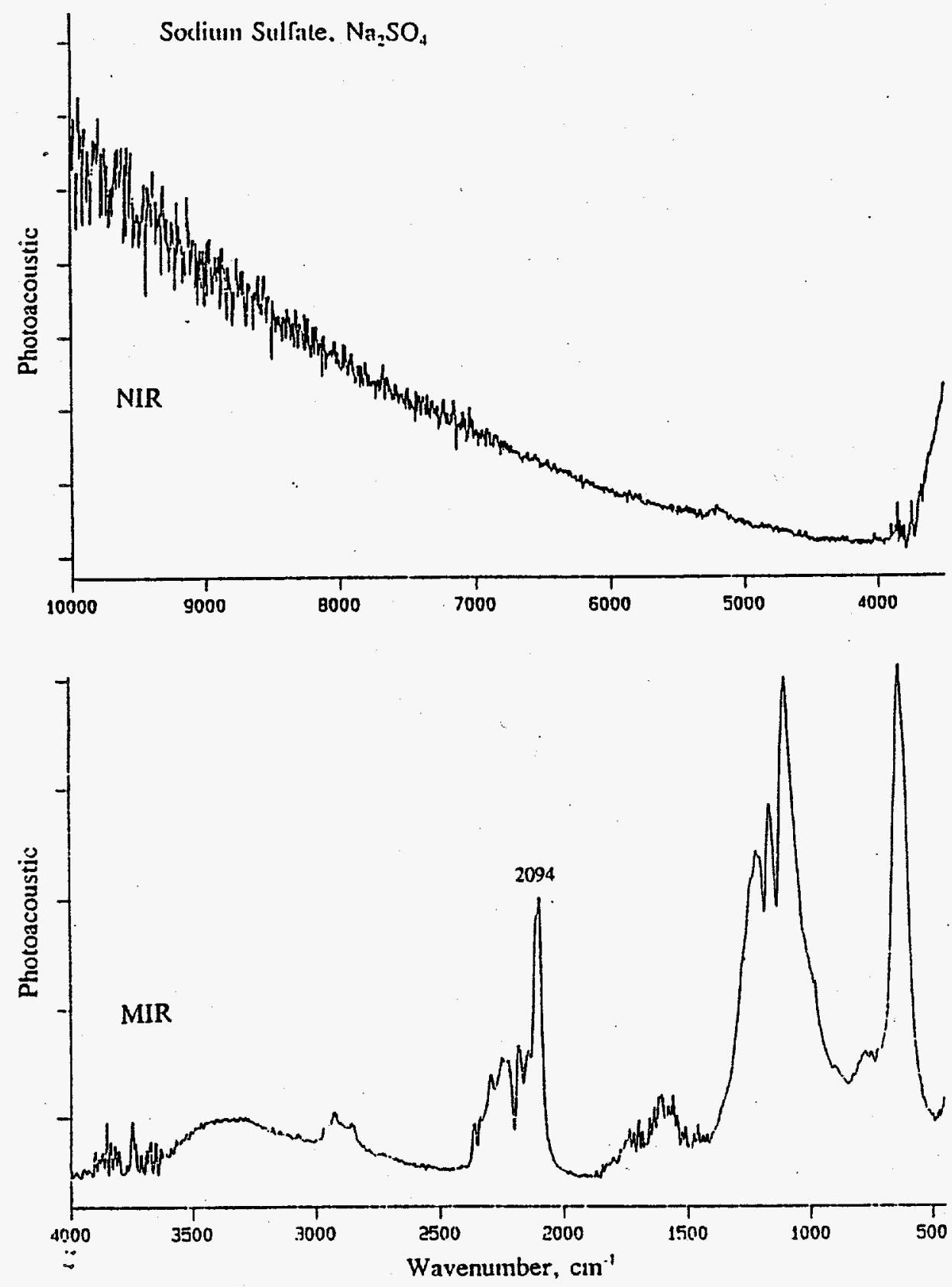

Figure 4-20. NIR and MIR Spectra of Sodium Sulfate Note: Photoacoustic Spectroscopy 

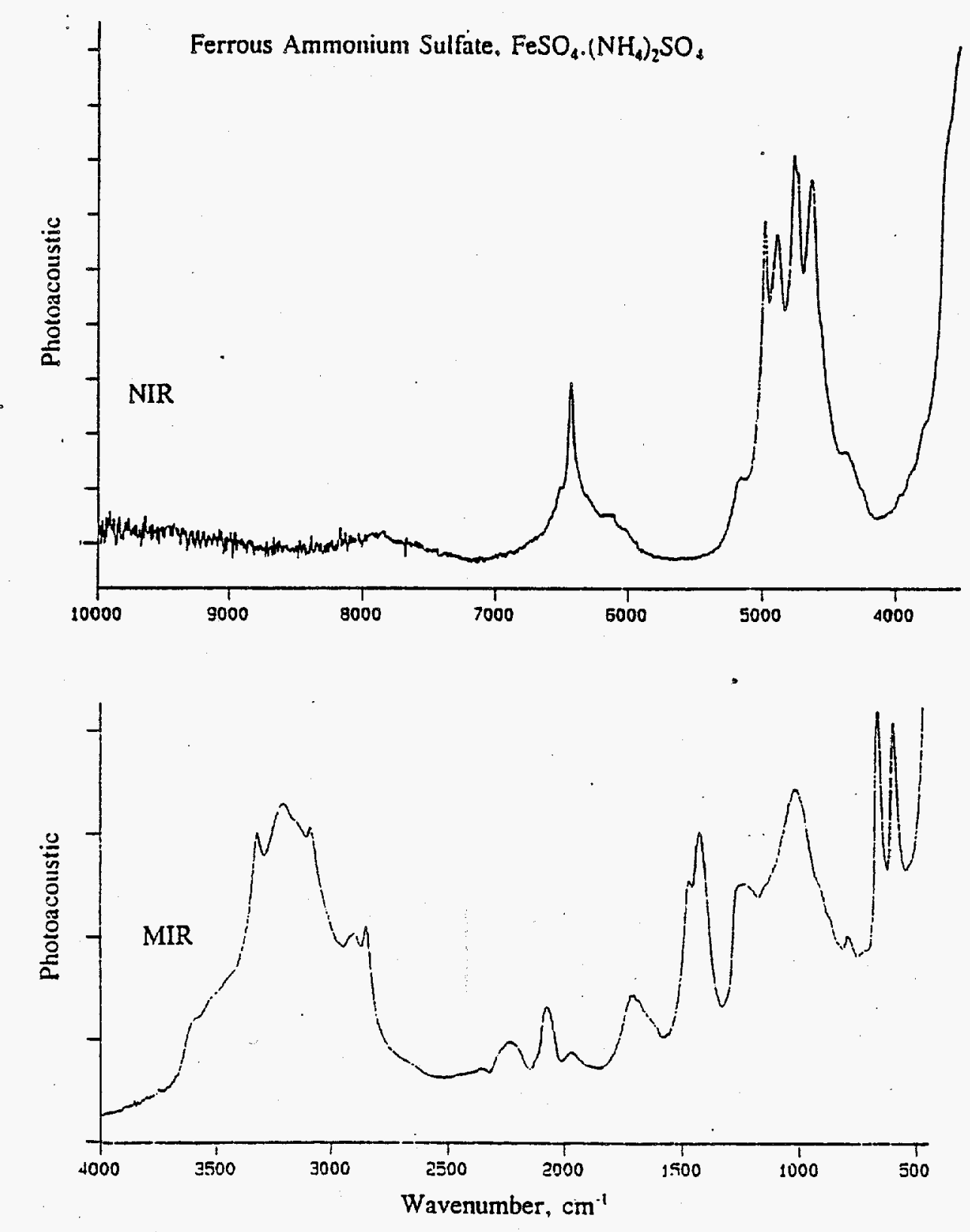

Figure 4-21. NIR and MIR Spectra of Ferrous Ammonium Sulfate Note: Photoacoustic Spectroscopy 
WHC-SD-WM-TI-664, Rev. 0
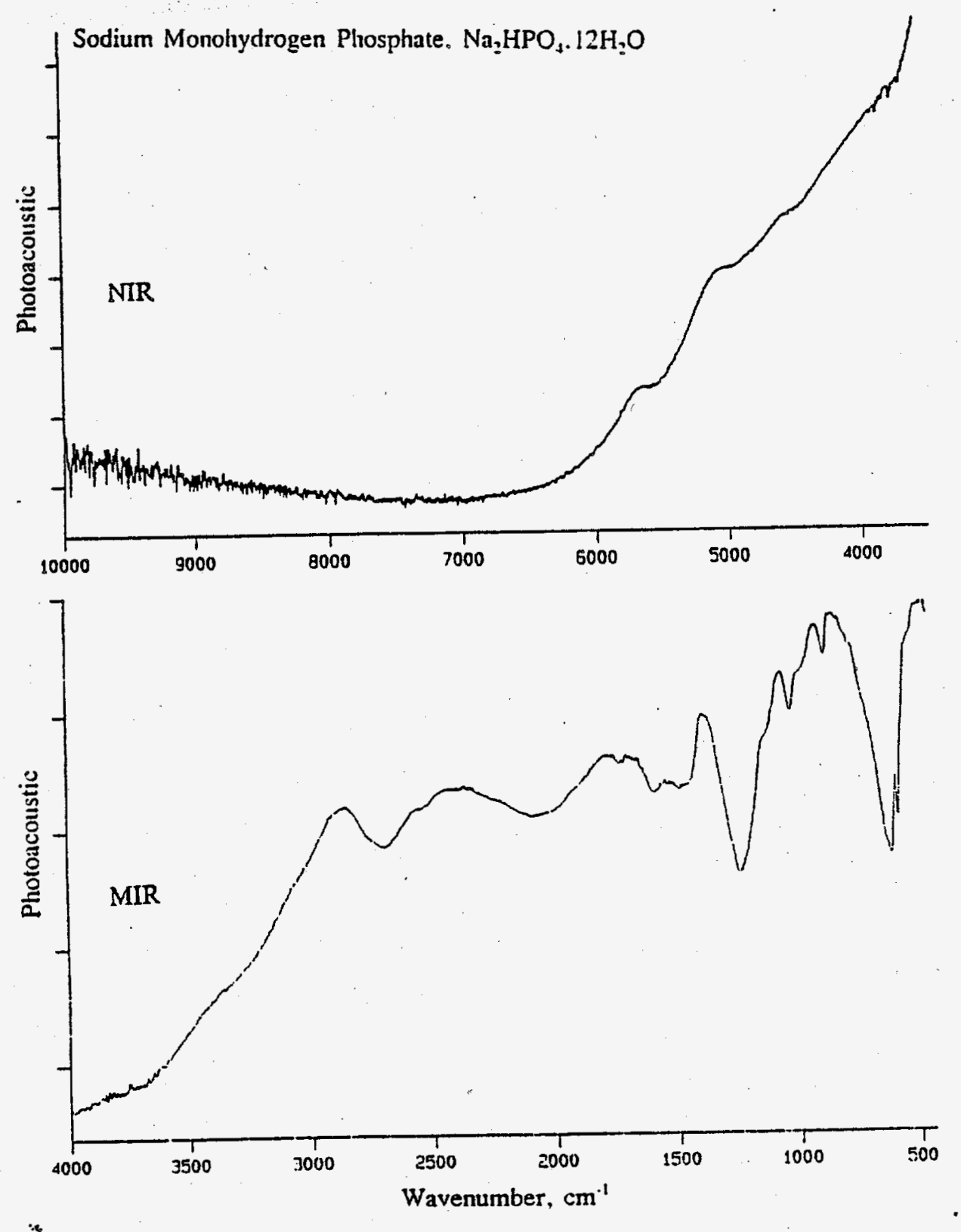

Figure 4-22. NIR and MIR Spectra of Sodium Monohydrogen Phosphate Note: Photoacoustic Spectroscopy 
WHC-SD-WM-TI-664, Rev. 0
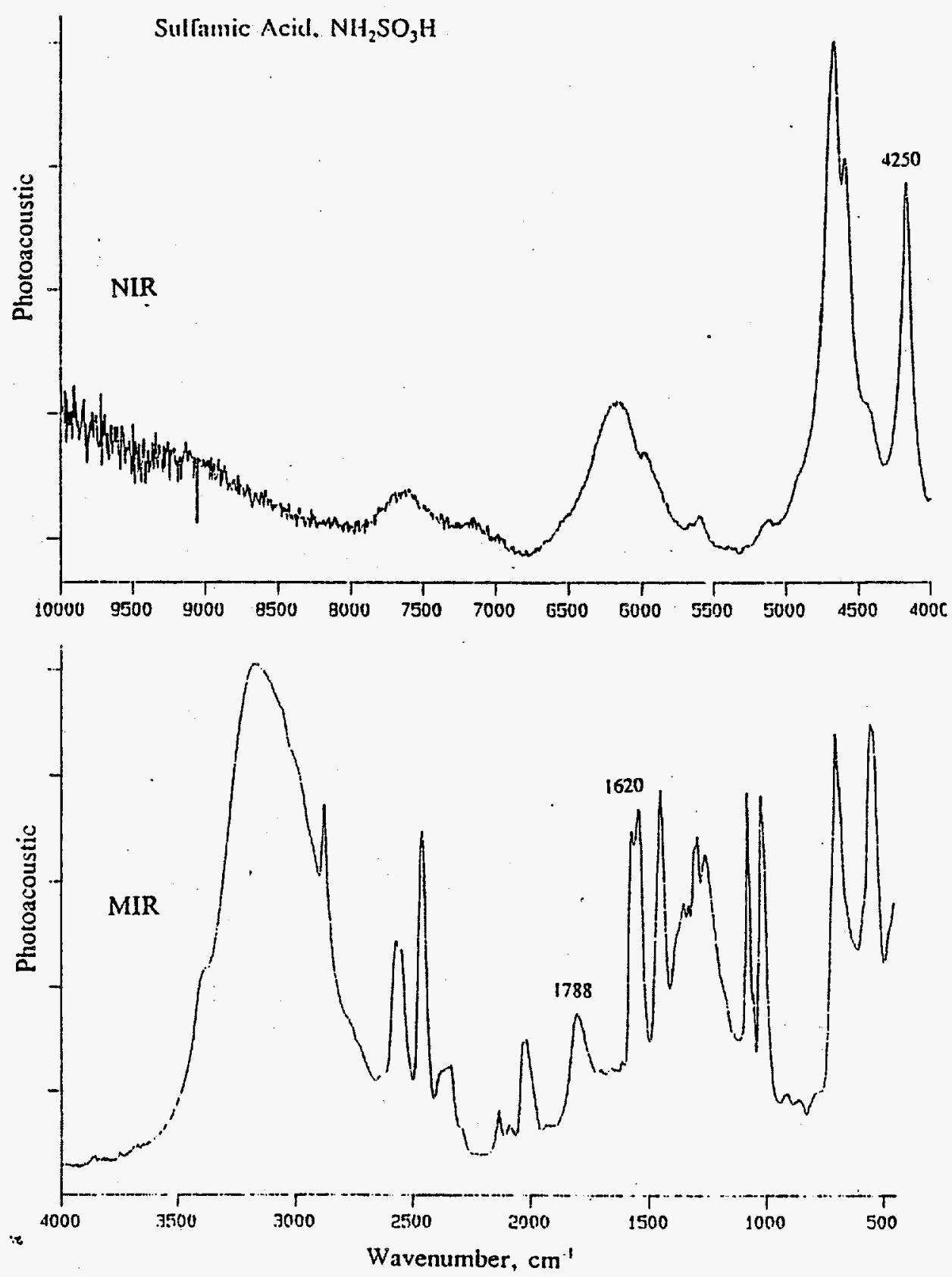

Figure 4-23. NIR and MIR Spectra of Sulfamic Acid Note: Photoacoustic Spectroscopy 


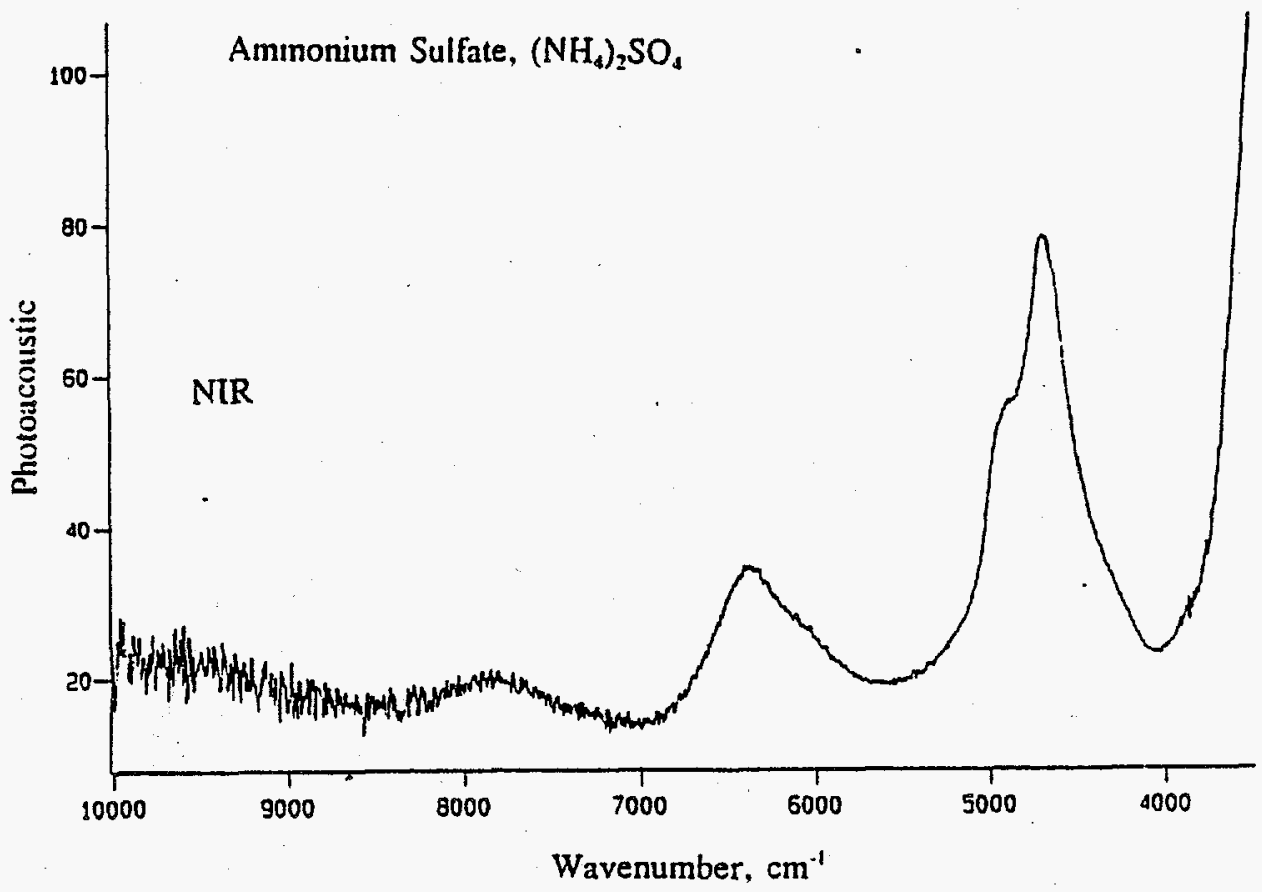

Figure 4-24. NIR Spectrum of Ammonium Sulfate Note: Photoacoustic Spectroscopy

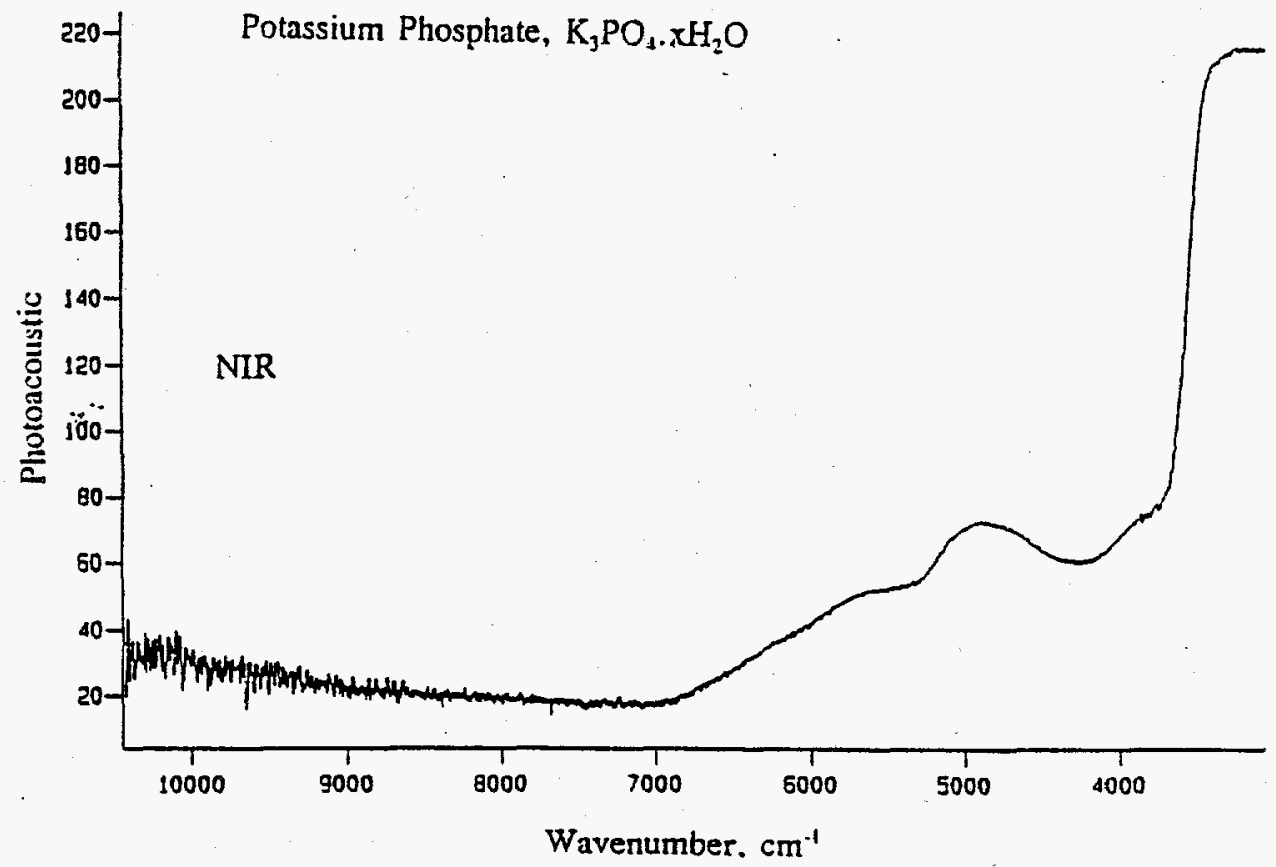

Figure 4-25. NIR Spectrum of Potassium Phosphate Note: Photoacoustic Spectroscopy 


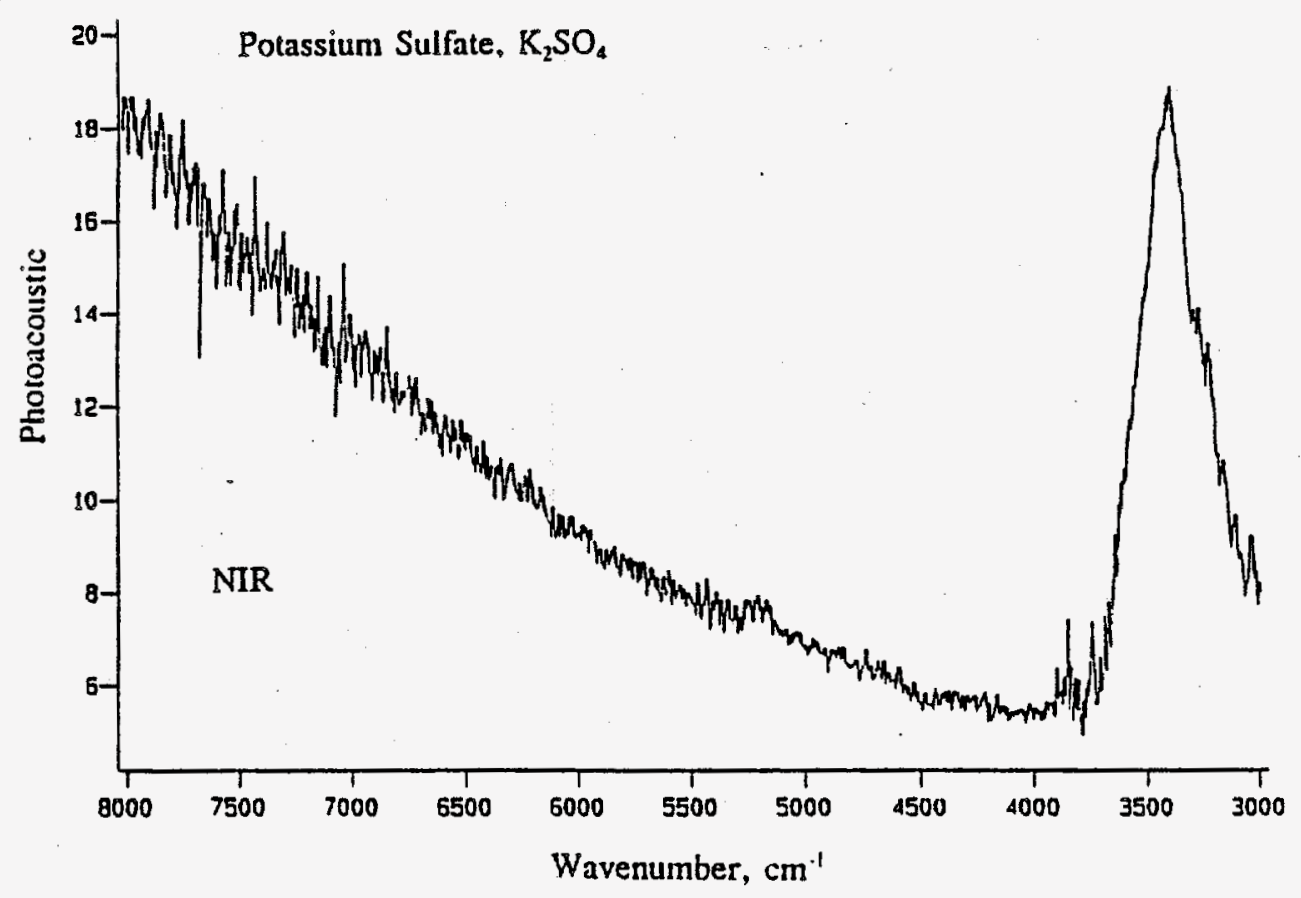

Figure 4-26. NIR Spectrum of Potassium Sulfate Note: Photoacoustic Spectroscopy

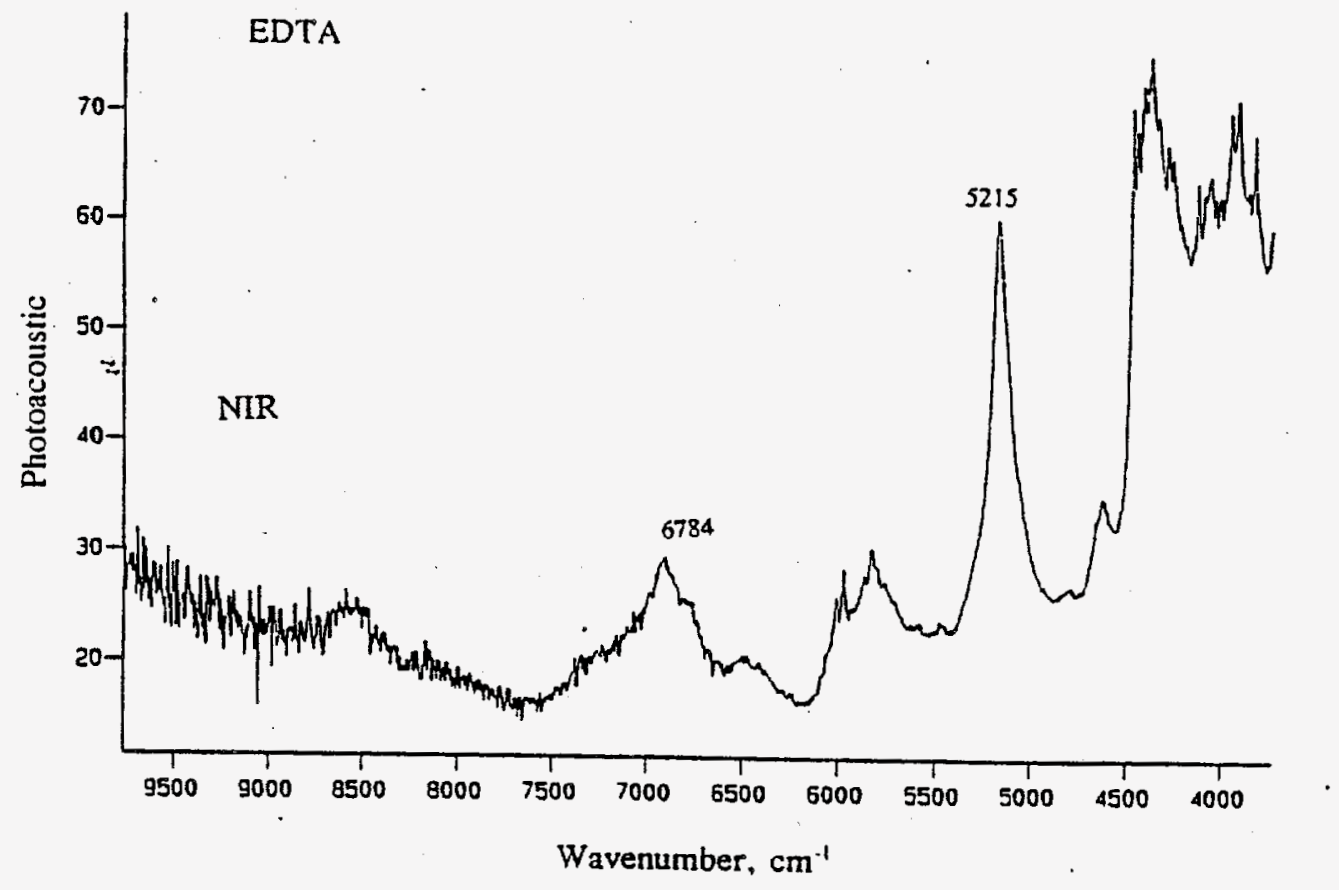

Figure 4-27. NIR Spectrum of EDTA

Note: Photoacoustic Spectroscopy 

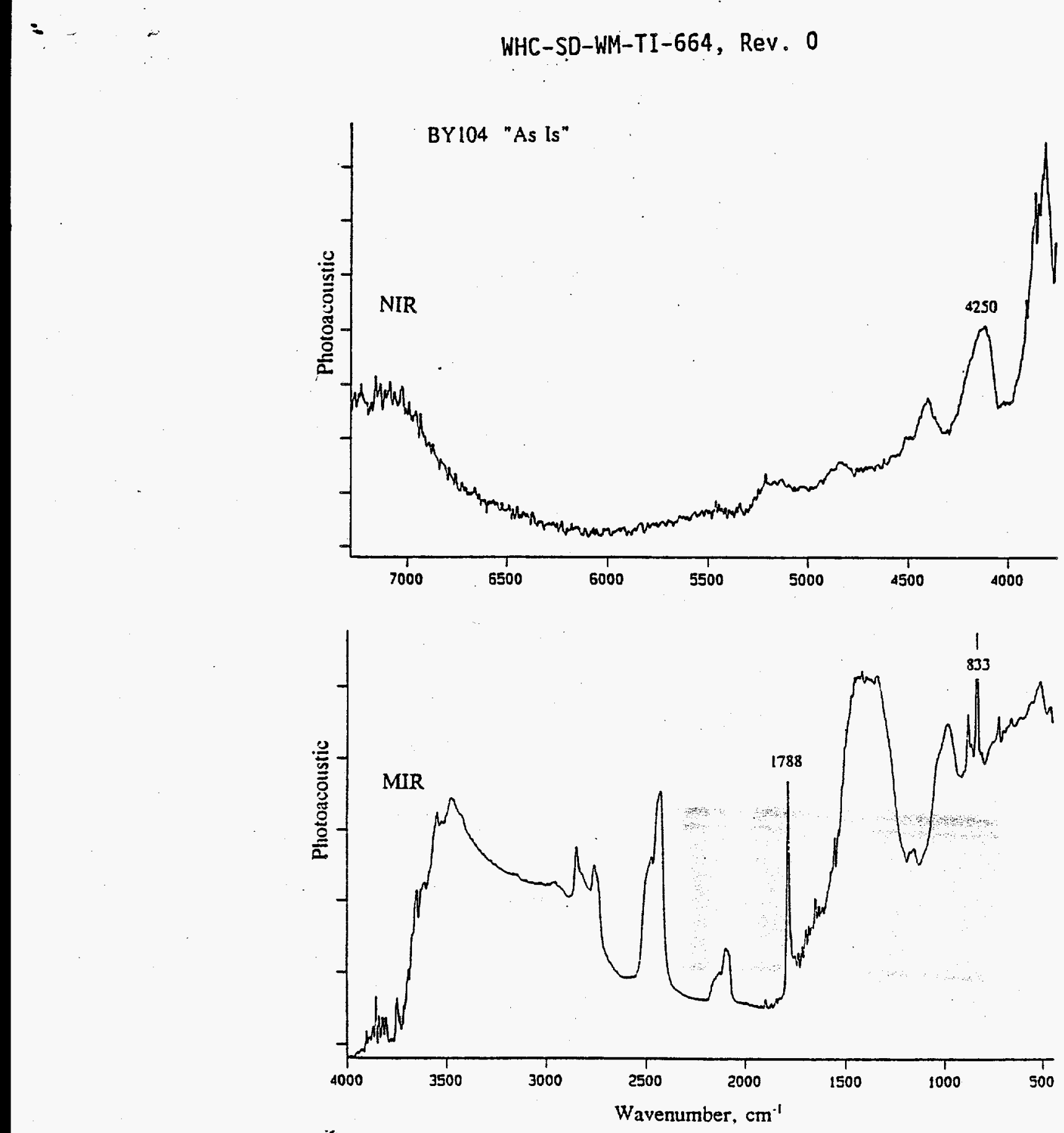

Figure 4-28. NIR and MIR Spectra of BY-104 Simulant Note: Photoacoustic Spectroscopy 

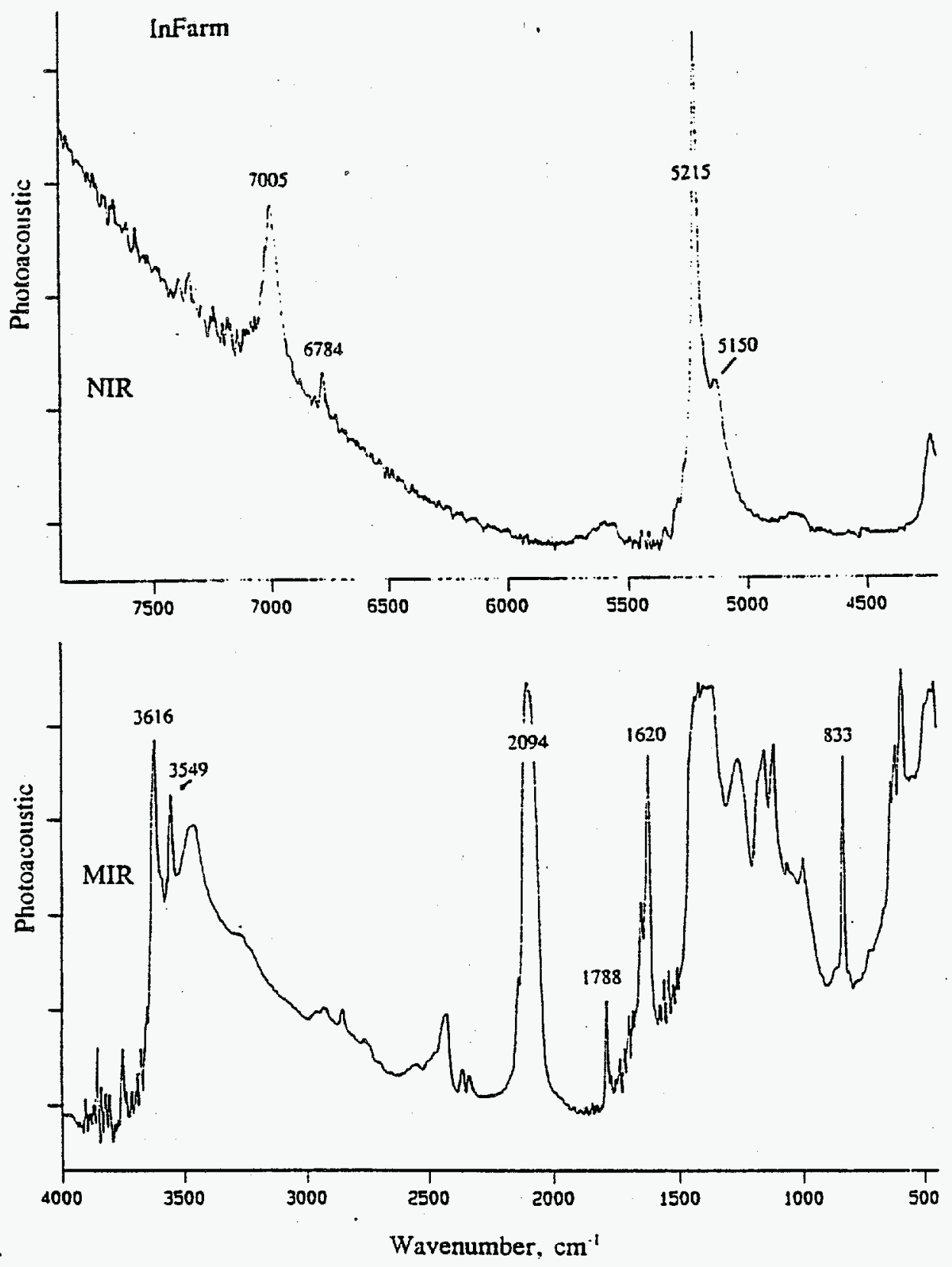

$\therefore$

Figure 4-29. NIR and MIR Spectra of In Farm Simulant Note: Photoacoustic Spectroscopy 

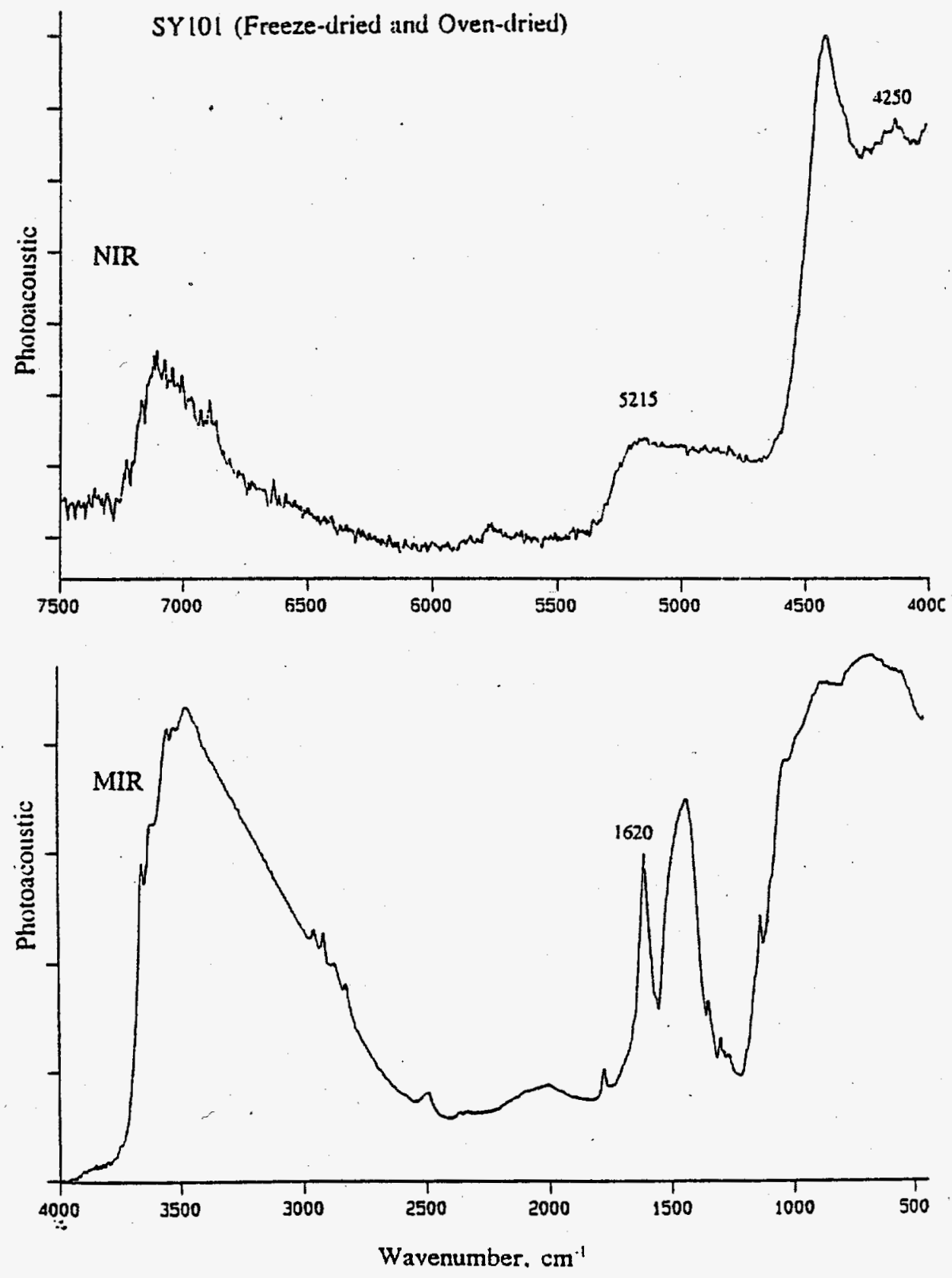

Figure 4-30. NIR and MIR Spectra of SY-10I Simulant Note: Photoacoustic Spectroscopy 

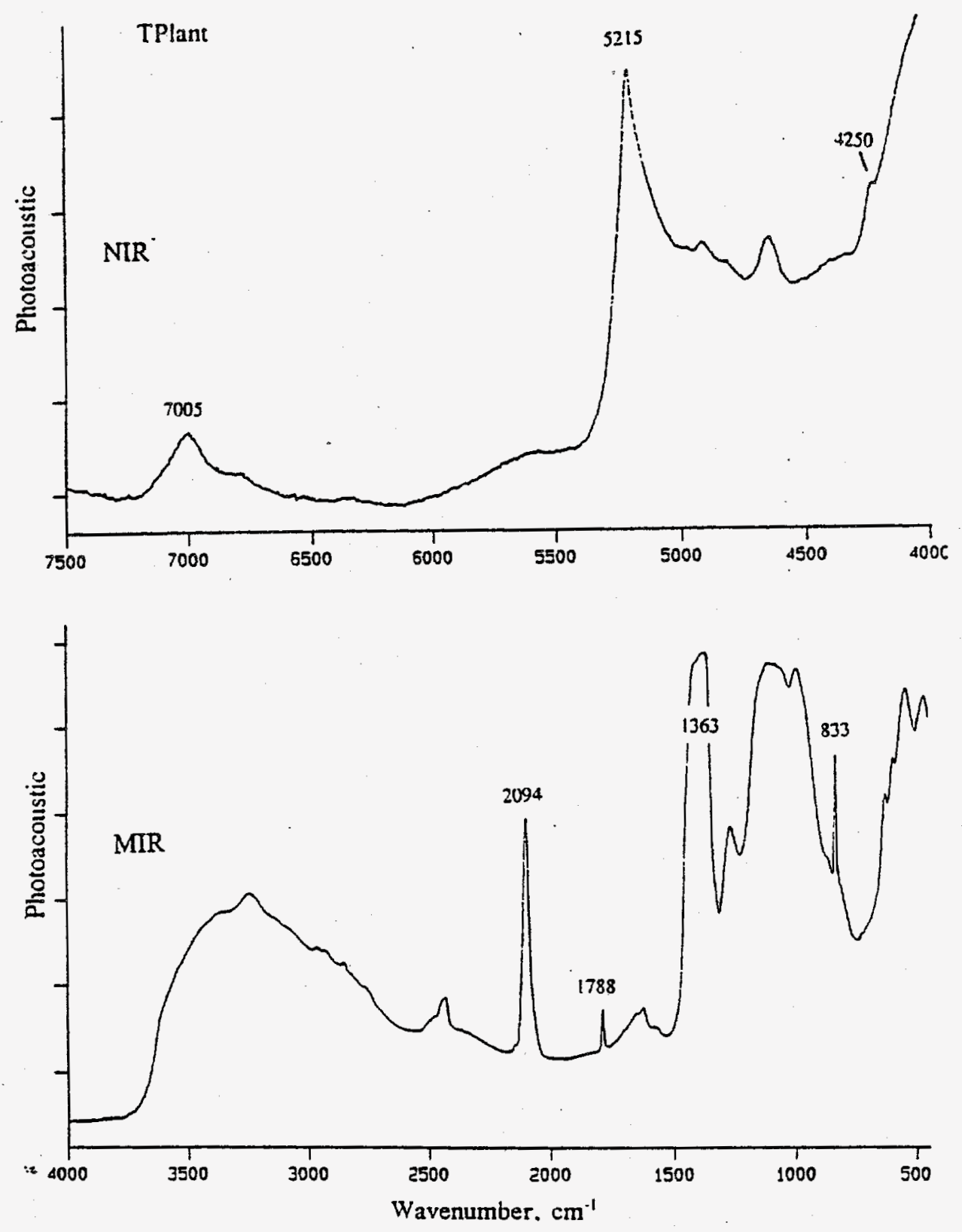

Figure 4-31. NIR and MIR Spectra of $T$ Plant Simulant Note: Photoacoustic Spectroscopy 

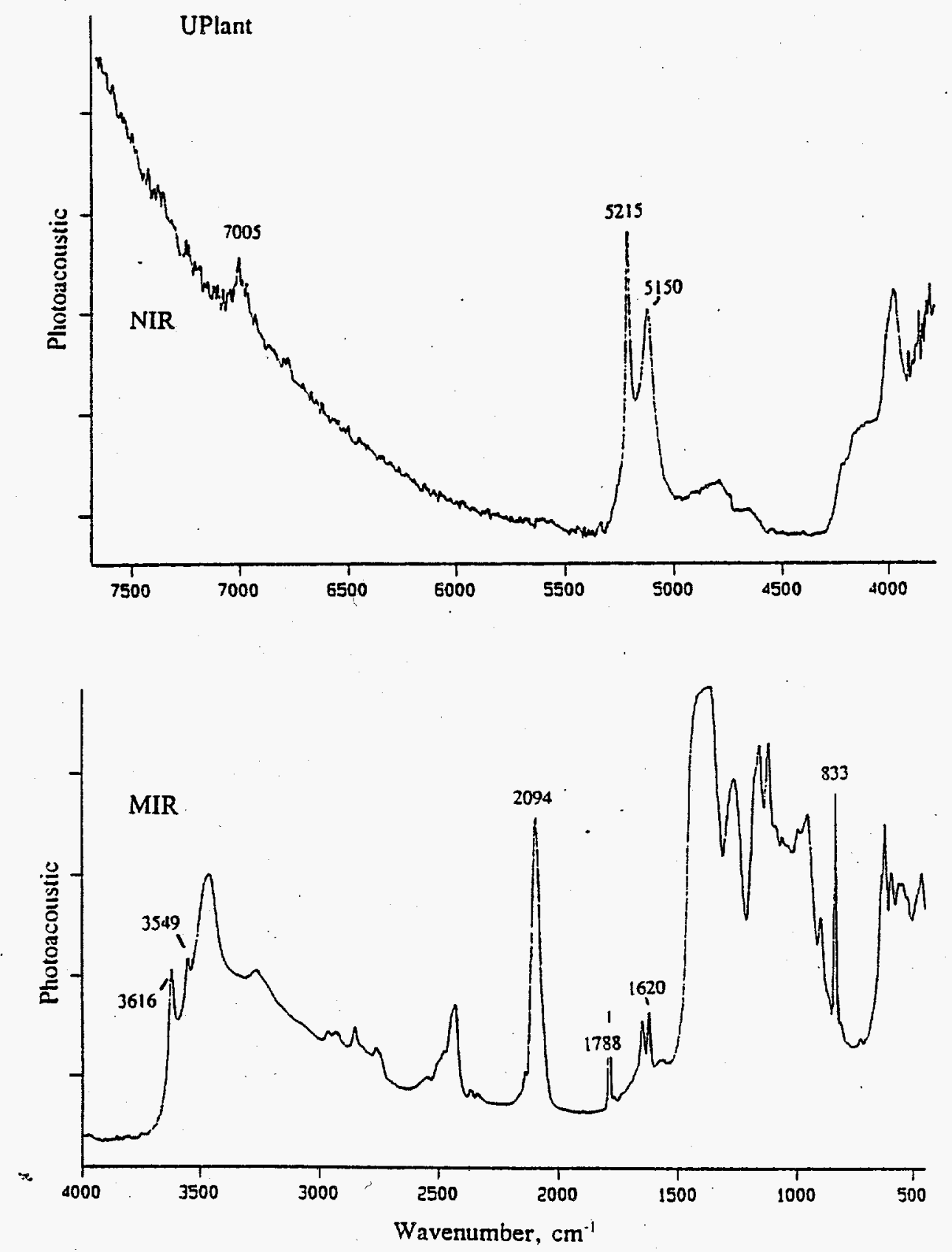

Figure 4-32. NIR and MIR Spectra of U Plant Simulant Note: Photoacoustic Spectroscopy 
WHC-SD-WM-TI-664, Rev. 0

\subsection{CONCLUSION}

The near-infrared (NIR) region of the infrared spectrum is the region of choice for the measurement of moisture in simulated waste. Differentiation of the NIR water spectrum, as a preprocessing step, will improve the analytical result. Both NIR and MIR spectra must be collected to measure ferrocyanide species unambiguously and accurately. For ease of sample handling and cleanup, as well as potential for field or tank deployment, the FTIR-Fiber optics system using diffuse/specular reflectance configuration is preferred over the attenuated total reflectance-evanescent field absorbance sensing method. More work, however, is needed to test whether the intensities of the combination band of water at $5215 \mathrm{~cm}^{-1}$ and the first overtone of the cyanide band at $4250 \mathrm{~cm}^{-1}$ can be utilized to measure water and ferrocyanide in actual wastes simultaneously. There is a good probability that free- and combined water can also be calculated from the water bands in the NIR and MIR spectral regions. The other two methods, modular transfer optics and photoacoustic spectroscopy, may be used as backup systems and for validation of the fiber optic data. 
WHC-SD-WM-TI-664, Rev. 0

\subsection{REFERENCES}

1. Getting Started Manua1, 1993, Bio-Rad/Digilab Division, Cambridge, MA., No. 091-0622C p.4.

2. Dittmar, R. M., J. L. Chao, and R. A. Palmer, 1991, "Photoacoustic Depth Profiling of Polymer Laminates by Step-Scan Fourier Transform Infrared Spectroscopy," App1. Spectrosc., 45, p.1104.

3. McClelland, J. F., R. W. Jones, S. Luo, and L. M. Seaverson, 1992, "A Practical Guide to FTIR Photoacoustic Spectroscopy," Center for Advanced Technology Development and Ames Laboratory, Iowa State University, Ames, Iowa.

4. System 2000 FT-IR User's Manual, 1992, Perkin Elmer Limited, Beaconsfield, U. K., pp.1-5 to 1-6.

5. Rebagay, T. V., D. A. Dodd, D. W. Jeppson, L. L. Lockrem, and G. R. Blewett, 1994; "In Situ Chemical Characterization of Waste Sludges Using FTIR-Based Fiber Optic Sensors, "WHC-SA-1987-Fp, Westinghouse Hanford Company, Richland, Washington.

6. Rosencweig, A., 1980, "Photoacoustic and Photoacoustic Spectroscopy," Wiley, New York, Chap. 9, pp.93-98. 